See discussions, stats, and author profiles for this publication at: https://www.researchgate.net/publication/323068542

Depth-averaged Lattice Boltzmann and Finite Element methods for singlephase flows in fractures with obstacles

Preprint · February 2018

CITATIONS

0

3 authors, including:

(3) Marcin Dabrowski

Państwowy Instytut Geologiczny

74 PUBLICATIONS 495 CITATIONS

SEE PROFILE

Some of the authors of this publication are also working on these related projects:

Project TCLB - the open source templated CUDA lattice Boltzmann solver View project

Project ShaleMech View project
READS

96

Michał Dzikowski

Państwowy Instytut Geologiczny

3 PUBlications 2 CITATIONS

SEE PROFILE 


\title{
Depth-averaged Lattice Boltzmann and Finite Element methods for single-phase flows in fractures with obstacles
}

\author{
3 M. Dzikowski ${ }^{\mathrm{a}, *}$, L. Jasinski ${ }^{\mathrm{a}}$, M. Dabrowski ${ }^{\mathrm{a}, \mathrm{b}}$ \\ ${ }^{a}$ Computational Geology Laboratory, Polish Geological Institute - National Research Institute, Wroclaw, Poland \\ ${ }^{b}$ Physics of Geological Processes, University of Oslo, Norway
}

\section{Abstract}

We use Lattice Boltzman Method (LBM) MRT and Cumulant schemes to study the performance and accuracy of single-phase flow modeling for propped fractures. The simulations are run using both the two- and three-dimensional Stokes equations, and a 2.5D Stokes-Brinkman approximate model. The LBM results are validated against Finite Element Method (FEM) simulations and an analytical solution to the Stokes-Brinkman flow around an isolated circular obstacle. Both LBM and FEM 2.5D Stokes-Brinkman models are able to reproduce the analytical solution for an isolated circular obstacle. In the case of 2D Stokes and 2.5D Stokes-Brinkman models, the differences between the extrapolated fracture permeabilities obtained with LBM and FEM simulations for fractures with multiple obstacles are below $1 \%$. The differences between the fracture permeabilities computed using 3D Stokes LBM and FEM simulations are below $8 \%$. The differences between the 3D Stokes and 2.5 Stokes-Brinkman results are less than $7 \%$ for FEM study, and $8 \%$ for the LBM case. The velocity perturbations that are introduced around the obstacles are not fully captured by the parabolic velocity profile inherent to the 2.5D StokesBrinkman model.

7 Keywords: FEM, LBM, fracture flow, Brinkman approximation

\section{Introduction}

Hydraulic fracturing is widely used in oil \& gas industry to improve hydrocarbons extraction from low-permeability matrix rocks such as shales [1]. It is also practiced to enhance heat extraction from sedimentary [2] and crystalline rocks [3]. Propping agents such as sand grains are injected into hydraulic fractures to counteract the mechanical operation of high wall pressures, which tend to reduce fracture aperture. The achieved distribution of proppant grains may have a large impact on the technological flows, which control transport processes in the hydraulic fractures and determine the exchange surface between the fracture network and the surrounding, low-permeability rock matrix. Fracture wall asperities play the role of proppant grains and maintain the aperture in natural rock fractures, which are dominant fluid pathways at great depths. The complex flow phenomena in propped fractures and their effective transport properties have been extensively investigated using lab experiments [4, 5], field observations [6], and numerical simulations [4]. Flow through apertures with cylindrical obstacles is also relevant to engineering applications such as micropillar arrays of cylinders used in Lab-on-a-chip systems for high performance liquid chromatography [7].

\footnotetext{
* Corresponding author

Email address: mjdzikowski@gmail.com (M. Dzikowski)
} 
The Lattice Boltzmann Method (LBM) is a weakly-compressible numerical method conceptually based on statistical thermodynamics and Boltzmann equation. The method is most commonly used to solve the Navier-Stokes equation, but it was successfully applied to problems that range from the Poisson or Poison-Boltzmann equations [8,9], through the Nerst-Planck [10] to plain convection equations [11]. The performance of LBM for incompressible flows range from proper path to approach low Mach number limit [12], development of general [13] and axisymmetric[14] schemes specifically for incompressible flows, and investigation of numerical techniques meant to avoid truncation error accumulation [15]. This work is concentrated on structured grid based LBM, but flow properties of random domains were also investigated using an unstructured grid variant of the method [16].

LBM is a relatively new method $[17,18,19]$ and attracts an increasing amount of attention, which has lead to its rapid development in recent years. In Earth Sciences Lattice Boltzmann Method has been used used in permeability calculation, for both single and multiple phase problems. The method itself historically orgins from cellular automaton fluid models (lattice automata, LCGA models [20]). Robustness of the lattice-based models for permeability computations was foreseen in the method early days [21]. Evolution of the modern formulation of the LBM [22, 23] led to permeability studies of random medium [24], including natural one [25]. Recent studies includes digital rock physics benchmarking study[26], where 3D tomography images where used to estimate permeability using LBM, among other rock properties calculated by using other methods. Porous media flows in general (especially multiphase or multiphysics cases) are one of the application areas that is often listed as well suited for LBM [27]. Prediction of pressure drop in such domains for various regimes were studied in a number of papers, including detailed experimental verifications using water tunnel and $\mu$-CT imaging [28]. Numerical investigation employing Lattice Boltzmann Method include, but are not limited to, artificially generated fibrous geometry [29], nanoscale simulations [30, 31] in low Knudsen number regime, investigations of scanned and generated geometries [32, 28, 33].

The Finite Element Method (FEM) is a well established numerical method for discretizing the weak integral formulation of partial differential equations. The method was originally developed for complex engineering problems of engineering applications in structural analysis, but it has been extended to a wide range of problems of mathematical physics. Using FEM for the incompressible Stokes problem requires some tailored approaches such as the mixed formulation [34] and dedicated solution schemes [35]. In Earth Sciences, FEM has been extensively used to study various deformation problems in heterogeneous media such as rock folding [36, 37, 38], earth mantle convection [39, 40], shear zone dynamics [41, 42], and particle suspension flows [43, 44]. FEM simulations have been also developed for purely hydrodynamic problems in geology. Most simulations are performed in the framework of upscaled, Darcy-type models for problems such as groundwater flow [45, 46], fracture and fracture network flow [47], multiphase flow in fractured porous media [48, 49], thermal and thermohaline convection in porous media $[50,51,52,53]$, and hydrocarbon migration $[54,55,56]$. With the advent of computer power and numerical method development, direct numerical simulations of single- and multiphase flow problems in fractures [57], porous matrix [58, 59,60], and dual-porosity media such as karst reservoirs [61,62], arise nowadays.

Direct numerical simulations of three dimensional fluid flows are computationally expensive and reduced-order, 2.5D numerical models have been proposed for flow through thin, planewalled domains. Holme and Rothman [63] presented a LBM study of miscible two-phase flows in a Hele-Shaw cell, including the effects of viscous drag due to the no-slip boundary conditions at the plates. Flekkøy et al. [64] used a LBM model for the flow and tracer transport in the central layer of a Hele-Shaw cell. The viscous drag effect was included by using a two-dimensional Brinkman-type equation for the mid-plane velocity, which was derived by assuming a parabolic 
velocity profile across the cell. Using an analytical solution for a rectangular channel as a benchmark, the error due to the approximation was estimated at less than $10 \%$ and the authors claimed that the simulations are expected to overestimate viscous drag close to the sidewalls. Clague et al. [65] used a three-dimensional LBM to study hydraulic permeability of bounded and unbounded fibrous media, with either ordered and disordered structure. The numerical results for the bounded disordered media were shown to be in a good agreement with the theoretical predicitions of Tsay and Weinbaum [66], who developed an effective medium approach to the problem based on a Brinkman-type equation derived by an in-plane rather than depth averaging.

Depth-averaging is often used to tread viscous coupling due to the plates in analytical studies of the flow in Hele-Shaw cells. For example, Fernandez et al. [67] presented a linear stability analysis of viscous fingering inside the Hele-Shaw cell based on an approximate two-dimensional Brinkman equation for the depth-averaged velocity. An LBM approach to viscous fingering in Hele-Shaw cells based on depth-averaging was used by Grosfils et al. [68]. Venturoli and Boek [69] used the same method in LBM simulations to analyze single phase flow in pseudo-2D capillary network models. Single and multiphase flow properties of realistic rock geometries were studied in another work of Boek and Venturoli [32]. Works by Hale et al. [7] and other by Hong et al. [70] are dedicated to multiphase flow in Hele-Shaw cell, mainly capillary action for thin domain with boundaries. The depth-averaged Stokes-Brinkman equation was used by Horgue et al. [71] and compared with experimental results for two-phase flows in the domain with cylindrical obstacles. Nagel et al. [72] used Boundary Element Method to solve the 2.5D Stokes-Brinkman model for a deformable droplet in a microfluidic flow in shallow channel. Laleian et al. [73] studied the accuracy of the depth-averaged 2.5D model for microfluidic devices with variable aperture. The computational speedup up to 40 times with respect to $3 \mathrm{D}$ simulations was reported along with an accuracy of $10 \%$ in terms of permeability.

In the present work, we introduce a 2.5D Stokes-Brinkman fracture flow model into MILAMIN [74], a FEM-based incompressible Stokes solver programmed in MATLAB, and TCLB [75, 76], a Lattice Boltzmann method based CUDA GPU code for Navier-Stokes problems. The 2.5D codes were validated against an analytical solution for the Stokes-Brinkman flow around a single circular obstacle and cross-checked against each other for systems with multiple obstacles. Fully resolved solutions were also obtained using the $3 \mathrm{D}$ variants of the two codes and we present a systematic comparison between the fully resolved 3D and the reduced 2.5D solutions to evaluate the accuracy and robustness of the approximate 2.5D Stokes-Brinkman fracture flow model.

\subsection{Problem statement}

\subsubsection{Geometric model and boundary conditions}

We use randomly placed cylinders as a simplified geometric model of a propped fracture (fig. 1). During the hydraulic fracturing process high fluid pressure keeps fracture open, allowing for proppant grains to be carried by the fracturing fluid ("initial state" in fig. 1). When the process is finished and pressure is released, fracture closes, and grains embed partially into rock matrix ("under closing pressure" in fig. 1). Finally, we assume that after embedment, grains appear cylindrical within the final fracture aperture after embedment

A sketch of the computational geometry is presented in fig. 2 (sketch in the middle). The cylinders are monodisperse and their positions are generated using the Random Sequential Adsorption [77], an algorithm for generating random packings of non-overlaping discs. The maximal packing fraction that can be generated using the RSA algorithm is $\varphi=0.547 \pm 0.002$ [77]. We use our own RSA implementation, which can support both periodic and non-periodic domains. In some tests, we run flow simulations for randomly placed squares. The no slip boundary condition is used at the rims of the inclusions. 
The fluid flow is single-phase and it is forced in $x^{+}$direction using either a pressure difference $\Delta p$ imposed on domain boundaries or a uniform mass force $G$ (gravitational). For the pressure difference loading, the free slip (symmetry boundary in LBM) is set at the $y$ direction boundaries, and traction boundary condition (with imposed pressure) at the inlet and outlet boundaries $(x$ direction) according to the prescribed pressure gradient. In the case of gravitational forcing, the velocity vectors are set periodic at the corresponding bottom-top and left-right boundaries. The 3D simulations are only performed for the pressure difference forcing. The no-slip boundary condition at the fracture walls ( $z$ direction) is directly enforced in 3D models and implicitly incorporated into the 2.5D models. In this study we are concentrated on incompressible Stokes flow through the thin gap with obstacles. Different aspects of porous media flow in general, such as finite Knudsen number effect consideration are not subject of this study. Reader interested in such effect could find them in dedicated papers [78].

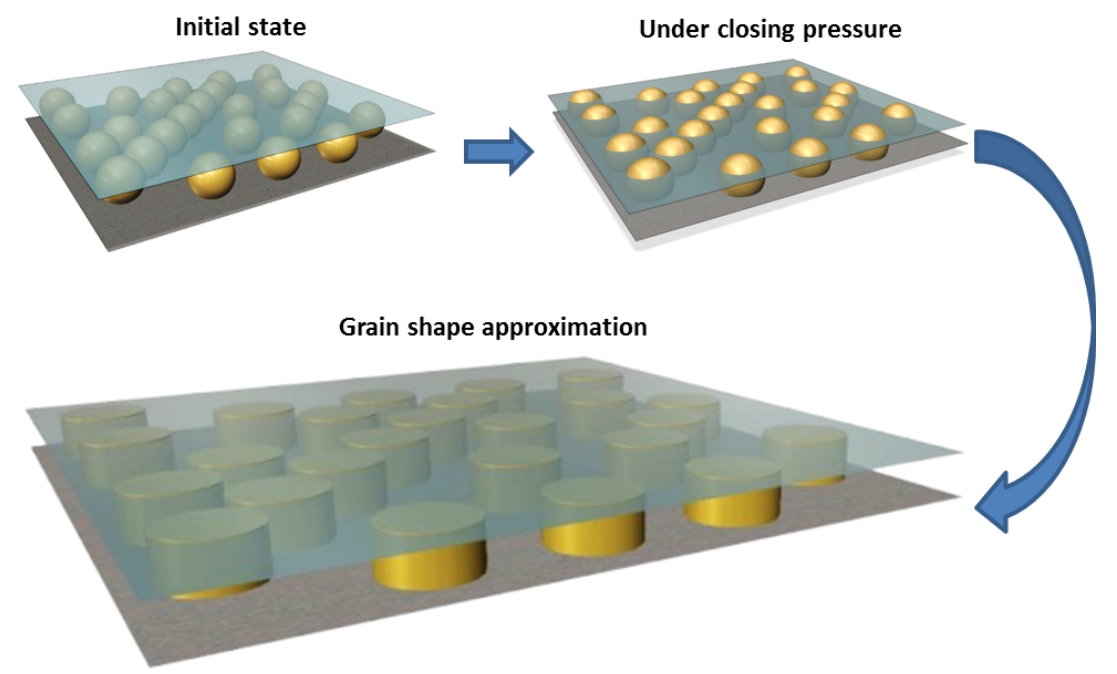

Figure 1: A simplified geometric model of the evolution of a propped fracture geometry. 

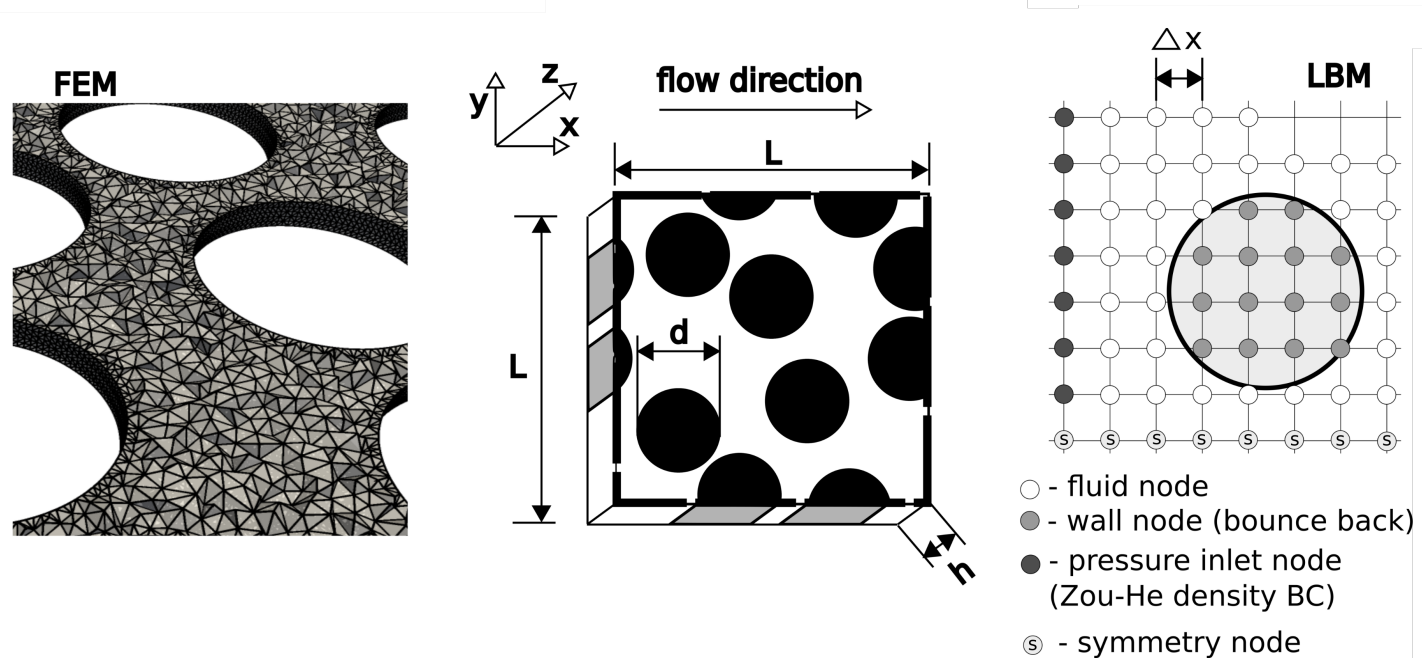

Figure 2: A) 3D unstructured mesh used in FEM simulations is shown.

B) Sketch of the geometrical model of the problem. The symbol $h$ denotes fracture aperture and $L$ denotes the edge length of the square domain.

C) Sketch of the LBM computational lattice is documented. Black circle represents an obstacle boundary.

\subsubsection{Computational meshes in LBM and FEM simulations}

Throughout the paper LBM refers to Lattice Boltzmann Method computations performed with TCLB code $[75,76]$ and FEM refers to computations performed with the MILAMIN Finite Element Method code [74]. The LBM simulations were carried out on structured grids with a uniform grid spacing $\Delta x=L / N X$, where $N X$ is the number of grid cells and $L$ denotes the edge length of the square domain. The FEM simulations were carried using unstructured meshes, and $\Delta x$ is defined as:

$$
\Delta x=P_{p} / N_{p}
$$

where $P_{p}$ is the inclusion perimeter and $N_{R}$ denotes the number of mesh segments along the inclusion perimeter. Sketch of the domain, and examples of tetrahedral mesh and uniform lattice used in computations are presented in fig. 2.

In LBM simulations, the model geometry is represented using a binary mask. We use the bounce-back boundary scheme to simulate no slip boundary schemes at the rims of the inclusions. The representation of the curvilinear boundaries of the inclusions could be improved by using other boundary schemes. However, the more accurate interpolation-based boundary conditions may be non-conservative, which could have detrimental effects in the studies of effective fracture permeability.

The binary representation of an arbitrary placed circle on a Cartesian grid is inherently inaccurate. The perimeter of a discretized circle is always equal to $4 d$ and the convergence of the area is not monotonic with increasing $N X$. These effects should be taken into account when considering properties that are strongly dependent on area or perimeter, especially when comparing solutions from simple bounce-back LBM simulations on structured grids and other numerical methods such as FEM on unstructured, body-fitting meshes. The introduced area representation error is typically small, but it may be a source of high area fraction errors if rounding of the positions and diameters is performed prior to marking cells that are inside each circle. On the other hand, if every discrete circle is masked using the actual floating point representations of the center location and diameter, the net area fraction error is greatly reduced. 
In our FEM simulations, we use either triangular or tetrahedral unstructured computational meshes fitting the rims of the inclusions. For 2D problems, we use Triangle mesh generator developed by J.R. Shewchuk [79] to obtain body-fitting triangular meshes. In 3D simulations, we use TetGen mesh generator developed by H. Si [80], which is capable of generating bodyfitting tetrahedral meshes for complex 3D input domains. Both mesh generators produce highquality meshes with an imposed limit on the minimum angle and are thus suitable for FEM computations. We use mtriangle and mtetgen routines from the MUTILS package [81] to facilitate data exchange between the mesh generators and MATLAB. To obtain mesh periodicity in both $x$ and $y$ directions, which is needed to impose periodic boundary conditions in our 2D FEM simulations, the periodic domain with inclusions is replicated, an auxiliary mesh is generated and the periodic boundary is set by searching for the shortest paths on the mesh segments between the neighboring corners of a unit cell.

\subsection{Numerical flow solvers}

Numerical simulations were carried out using self-tailored variants of the open source packages: TCLB (github.com/CFD-GO/TCLB)[75] and MILAMIN (www.milamin.org)[82]. The former is a Lattice Boltzmann package that uses CUDA technology to handle massively parallel computations on multiple GPUs and the latter package is a Matlab implementation of a FEM based incompressible Stokes solver for desktop computers and SMP machines.

\subsubsection{Lattice Boltzmann Method Navier-Stokes solver TCLB}

The Lattice Boltzmann Method is a cellular automaton based method for solving the NavierStokes equations. The method is derived from the Boltzmann equation, which governs the evolution of the particle distribution function in rarefied gases. In LBM, the original system of integro-differential Boltzmann equations is discretized in the phase space and supplemented by assumptions related to the collision integrals such as the Bhatnagar-Gross-Krook collision approximation (BGK) [83], and it converges to Navier-Stokes system in the limit of small Knudsen number (a detailed discussion of Boltzmann equation in the context of LBM and the Lattice Boltzmann method itself can be found in $[27,84]$ ). It was also demonstrated that both widely used method variants: single and multiple relaxation methods[85], can converge to the the solution of incompressible Navier-Stokes equation [12] in the limits of vanishing Mach number and finite Reynolds number. To avoid issues related to higher Knudsen numbers, in all simulations numerical Knudsen number was

$$
K \approx \frac{M a}{R e}=\frac{\nu}{c L}<0.06
$$

with numerical $R e=7-40$ and $M a<0.05$. LBM iteration scheme is explicit and the update rule is traditionally stated in terms of collision and streaming operations

$$
f_{j}\left(\mathbf{x}+\mathbf{e}_{j}, t+\Delta t\right)=f_{j}(\mathbf{x}, t)+\Omega_{j}+\Delta f_{j}
$$

where $\mathbf{x}$ is the position of a grid point, $\mathbf{e}_{j}$ denotes the vector pointing from the lattice grid point to its $j$ th neighbor (only nearest neighbors are considered here; the reader is referred to [27] for a description of different elements and lattice types in LBM ), $t$ is a discrete time, and $f_{j}$ is the probability density associated with the $j$ th velocity from the discrete set $\mathbf{c}_{j}=\mathbf{e}_{j} / \Delta t$. The term $\Omega_{j}$ denotes the collision operator. The LBM method is unsteady by its nature, but attempts were also made to avoid explicit time marching in the LBM schemes to obtain steady state solution. For example a matrix formulation presented by Bernashi et al. [86] where preconditioned iterative solvers are used to obtain steady state solution. Similar approach was also employed earlier in the Stokes regime [87]. 
The BGK approximation simplifies the collision operator to linear relaxation

$$
\Omega_{j}=\omega\left(f_{j}-f_{j}^{e q}\right)
$$

where $f_{j}^{e q}$ stands for the equilibrium distribution function and $1 / \omega$ is a relaxation time. Direct implementation of the (3) is called Single Relaxation Time and it is presented here only for reference. We choose the more stable Multiple Relaxation Time (MRT) [85] approach and recently developed Cumulant scheme [88](C) collision operators ${ }^{1}$. In both MRT and C methods, the advection (streaming) part of equation (2) is performed with the probability densities $f_{i}$, while the collision step is performed using some other variables. In the MRT variant of the LBM, the collision operator $\Omega_{i}$ is transformed to perform the relaxation step in the moments space. The transformed collision operator can be written as

$$
\Omega_{j}=\left(M^{-1}\right)_{i j}\left[\omega_{i}\left(M_{i j} f_{j}-m_{i}^{e q}\right)\right]
$$

where $M_{i j}$ is the transformation matrix, and $m_{j}$ is $j$-th moment (usually the first moments are macroscopic quantities such as momentum and density). For 2D simulation, we use

$$
M_{i j}=\left[\begin{array}{ccccccccc}
1 & 1 & 1 & 1 & 1 & 1 & 1 & 1 & 1 \\
0 & 1 & 0 & -1 & 0 & 1 & -1 & -1 & 1 \\
0 & 0 & 1 & 0 & -1 & 1 & 1 & -1 & -1 \\
-1 & 2 & -1 & 2 & -1 & 2 & 2 & 2 & 2 \\
-1 & -1 & 2 & -1 & 2 & 2 & 2 & 2 & 2 \\
0 & 0 & 0 & 0 & 0 & 3 & -3 & 3 & -3 \\
0 & 0 & -1 & 0 & 1 & 2 & 2 & -2 & -2 \\
0 & -1 & 0 & 1 & 0 & 2 & -2 & -2 & 2 \\
1 & -2 & -2 & -2 & -2 & 4 & 4 & 4 & 4
\end{array}\right], \omega_{j}=\left[\begin{array}{c}
0 \\
0 \\
0 \\
s_{2} \\
s_{2} \\
s_{2} \\
s_{3} \\
s_{3} \\
s_{4}
\end{array}\right]
$$

where $s_{2}=1 /\left(3 \nu+\frac{1}{2}\right), s_{3}=1 \frac{1}{3}$ and $s_{4}=0$. In the Cumulant approach by Geier et al. [88], the moments are further transformed non-linearly to cumulants.

The term $\Delta f_{j}$ corresponds to the body force. In the case of the MRT collision operator, the Exact Difference Method term [89, 90] was implemented:

$$
\Delta f_{j}=f^{e q}(\rho, \mathbf{u})-f^{e q}\left(\rho, \mathbf{u}+\frac{\mathbf{F}}{\rho}\right)
$$

where $\mathbf{F}$ denotes the body force (i.e. gravity). Similar approach (with $\mathbf{u}+\frac{\mathbf{F}}{\rho}$ used in collision operator directly, the "equilibrium shift" method) is often used in multiphase simulations [91, 92]. Macroscopic properties of the fluid, density $\rho$ and velocity $\mathbf{u}$, can be determined from:

$$
\begin{gathered}
\rho=\sum f_{j} \\
\rho \mathbf{u}=\sum \mathbf{e}_{j} f_{j}-\mathbf{F} \frac{\Delta t}{2}
\end{gathered}
$$

where the summation is taken over all discrete directions (nine for the D2Q9 element, nineteen for the D3Q19 element, see [27] for details). Implementation of the Cumulant collision operator

\footnotetext{
${ }^{1}$ Numerical code TCLB used in this paper is publicly available at https://github.com/CFD-GO/TCLB . Both MRT and Cumulant models implementations are available
} 
follows a similar approach, but dedicated to the Cumulant relaxation scheme [88]. For the cases involving inlet/outlet boundary conditions instead of gravitational forcing, we choose scheme proposed by Zou-He [93].

The LBM in a transient solver by nature and a steady state solution needs to be reached before the flow properties can be evaluated. For most cases we have performed at least $N_{t} / N_{x}=$ 400 iterations and ensured afterwards that observed parameters reached equilibrium. The only exceptions where $3 \mathrm{D}$ ones, where $N_{t} / N_{x}=100$.

\subsubsection{Finite Element Method Stokes solver MILAMIN}

MILAMIN [74] is an efficient, FEM-based incompressible Stokes solver programmed in MATLAB for large 2D problems on unstructured computational meshes. The code is based on the mixed formulation of FEM and the computations are performed using the LBB-stable CrouzeixRaviart seven-node triangular element with a conforming, quadratic velocity space enriched with a bubble shape function and a linear discontinuous pressure. The FEM discretization of the incompressible Stokes problem in MILAMIN results in an indefinite, symmetric system of linear equations:

$$
\left(\begin{array}{cc}
A+\kappa B^{T} M^{-1} B & B^{T} \\
B & 0
\end{array}\right)\left(\begin{array}{l}
v \\
p
\end{array}\right)=\left(\begin{array}{l}
f \\
g
\end{array}\right)
$$

where $v$ and $p$ denote correspondingly the discrete velocity and pressure, $A$ is the stiffness matrix, $B$ is the discrete divergence operator, $f$ and $g$ are the rigth-hand side terms taking into account both the Dirichlet and Neumann boundary conditions. Symbol $\kappa$ stands for the penalty factor in front of the $B^{T} M^{-1} B$ term due to the augmented Lagrangian method, in which $M$ denotes the pressure mass matrix. By performing a block Gaussian elimination of 4 , a symmetric, positivedefinite pressure Schur complement arises:

$$
B\left(A+\kappa B^{T} M^{-1} B\right)^{-1} B^{T} p=B\left(A+\kappa B^{T} M^{-1} B\right)^{-1} f-g
$$

In the original MILAMIN code, steepest descent iterations were run on the pressure Schur complement 5 to obtain the pressure field in the incompressible limit (the so-called Powell-Hestenes iterations). For a discontinuous pressure space, the inverse of the pressure mass matrix $M^{-1}$ can be computed on the element level. Thus, the inner matrix $A+\kappa B^{T} M^{-1} B$ remains sparse and it can be formed explicitly (the so-called static condensation). On the other hand, the pressure Schur complement is not computed explicitly. The inner matrix is symmetric and positive-definite and its Cholesky factor is computed and stored prior to pressure iterations. The forward and backward substitutions are applied to the factor to find the action of the inverse of the inner matrix during the iterations. In some later application of the MILAMIN code, the steepest descent iterations were replaced by conjugate gradient iterations preconditioned with the pressure mass matrix. The condition number of the outer system can be improved by increasing the penalty factor $\kappa$, which results in a decreased number of conjugate gradient iterations. However, too high penalty factor can result in an ill-conditioning of the inner system and, although the Cholesky factorization is a highly robust algorithm, numerical problems may arise for large systems. We use $\kappa=1000$ in most of the FEM simulations in this paper. Once the pressure is found, the velocity field can be recovered by computing

$$
v=\left(A+\kappa B^{T} M^{-1} B\right)\left(f-B^{T} p\right)
$$

The Cholesky factorization exhibits a non-linear scaling with increasing the number of degrees of freedom, both in terms of storage and computations. However, the nonlinearity is rather moderate in the case of $2 \mathrm{D}$ problems and, if an appropriate fill-in reducing reordering of the matrix 
is used, the approach can be successfully used for systems up to several dozens of millions of degrees of freedom. Due to a higher nodal connectivity, the nonlinear scaling of the operations is much more severe for 3D problems and, despite highly efficient, multi-threaded implementations of the Cholesky factorization, the approach is impractical for 3D problems exceeding a few hundred thousand of degrees of freedom. Instead, we use the minimal residual (minres) iterations directly on the indefinite system (5) with $\kappa$ set to 0 or a small value. The minres iterations are preconditioned by either a simple Jacobi or a two-level BPX type of preconditioner for the $A$ block and the pressure mass matrix for the remaining part. The heavy sparse matrix vector multiplications during the iterations are performed with a NUMA-aware routine from the MUTILS package [94], which ensures both a tailored blocked storage of the symmetric sparse matrix (only the lower part is stored) and an almost optimal memory bandwidth during the computations.

\subsection{Numerical accuracy of the TCLB and MILAMIN Stokes solvers in $2 D$ and $3 D$}

We study the numerical performance of the TCLB and MILAMIN solvers for an incompressible Stokes flow between cylindrical obstacles in 2D and in the complex aperture space of 3D synthetic analogous of the propped fractures. We focus our tests on the accuracy and convergence of the effective fracture permeability as a function of numerical resolution. The convergence and accuracy of the LBM solutions is also influenced by the accuracy of the geometrical representation of circular inclusions on a structured grid and the method used to impose boundary conditions. The performance of the MRT and Cumulant collision schemes is studied in the limit of incompressible flow $(M a \rightarrow 0)$ and in the Stokes flow limit $(R e \rightarrow 0)$. The pseudo-time iterations are run to achieve the steady state solution. We run systematic FEM simulations to obtain independent results directly in the Stokes limit, check the convergence and validate the LBM and FEM results against each other. Selected results for the extrapolated effective permeability are summarized in table 1.

\begin{tabular}{|c|c|c|c|c|c|c|c|c|}
\hline & Name & \# of. incl. & frac. & $H / R$ & $\dot{\vec{\Phi}}$ & forcing & $L / R$ & $K_{\infty} / R^{2} \cdot 10^{3}$ \\
\hline \multicolumn{9}{|c|}{ Periodic domain, 2D model } \\
\hline \multirow{3}{*}{ 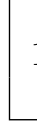 } & 2D, G, MRT, periodic, n100 & \multirow{3}{*}{100} & \multirow{3}{*}{0.45} & \multirow{3}{*}{ - } & \multirow{3}{*}{$\mathrm{Y}$} & \multirow{3}{*}{$G$} & \multirow{3}{*}{26.46} & 19.475 \\
\hline & 2D, G, C, periodic, n100 & & & & & & & 19.55 \\
\hline & 2D, G, FEM, periodic, n100 & & & & & & & 19.462 \\
\hline \multicolumn{9}{|c|}{ Periodic domain, 2D model, rectangular obstacles } \\
\hline \multirow{2}{*}{2} & 2D, G, MRT, periodic, n100,R & \multirow{2}{*}{100} & \multirow{2}{*}{0.14} & \multirow[b]{2}{*}{ - } & \multirow{2}{*}{$\mathrm{Y}$} & \multirow[b]{2}{*}{$G$} & \multirow{2}{*}{21.33} & 62.4 \\
\hline & 2D, G, FEM, periodic, n100,R & & & & & & & 63.5 \\
\hline \multicolumn{9}{|c|}{ Non-periodic domain, 2D \& 3D model } \\
\hline \multirow{3}{*}{3} & 2D, MRT, nonperiodic, n128 & \multirow{5}{*}{128} & \multirow{5}{*}{0.5} & \multirow{3}{*}{ - } & \multirow{5}{*}{$\mathrm{N}$} & \multirow{5}{*}{$\Delta P$} & \multirow{5}{*}{28.33} & 5.832 \\
\hline & 2D, C, nonperiodic, n128 & & & & & & & 5.843 \\
\hline & 2D, FEM, nonperiodic, n128 & & & & & & & 5.78 \\
\hline \multirow{2}{*}{6} & 3D, C, nonperiodic, n128 & & & \multirow{2}{*}{0.567} & & & & 2.15 \\
\hline & 3D, FEM, nonperiodic, n128 & & & & & & & 2.31 \\
\hline
\end{tabular}

Table 1: A comparison of the extrapolated effective permeability obtained with LBM and FEM for various model geometries and loading scenarios. "C" refers to solutions obtained using Cumulant LBM variant, "MRT" denotes Multiple Relaxation Time variant of LBM, see 1.2.1. "FEM" refers to results obtained using finite element approach. "G" are cases driven by gravity acceleration and " $\mathrm{R}$ " are cases with rectangular obstacles( where $L / R$ is equal to $L / a$, where $a$ is a square edge length). 


\subsection{1. $T C L B$}

For each numerical resolution $\Delta x$, the effective permeability $K$ was evaluated by averaging the net flow discharges in constant $x$ cross-sections, which were computed using the Simpson's integration rule. During the velocity field integration, we consider the bounce-back boundary condition, which implies the zero-velocity boundary mid-link. We calculate the relative error with respect to an approximate limiting solution $K_{\infty}$, obtained by fitting $K_{\infty}+b(\Delta x)^{c}$ to the effective permeability for the three highest resolution data points.

Firstly, we consider numerical convergence of both collision schemes in various combinations of flow forcing and obstacles shape. In fig. 3 those results are presented. All three two-dimensional periodic cases (flow forced by gravity): MRT and Cumulant, both with circular obstacles, and MRT with square obstacles (denoted R) have similar convergence rate. However, the Cumulant scheme (crosses) converges at slightly lower rate. In the case of nonperiodic setups (driven by pressure difference ) convergence rate of MRT and Cumulant scheme behaves similarly, but with higher absolute value of the error for all collision methods. The only clearly different curve (straight triangles) belong to 3D simulations with convergence rate approximately twice higher than in $2 \mathrm{D}$ cases. Relative error is calculated with respect to approximate infinite resolution result, extrapolated from obtained data. The 3D case have relatively low highest resolution (1024 nodes per $L$, instead of 4096 used in 2D cases), which might influence the estimation of the error.

The effects related to the accuracy of the geometry representation can be elucidated by comparing the results for cylindrical and rectangular obstacles, which are also presented in fig. 3 . Data presented indicate that obstacle shape does not have significant influence on the convergence, but it seems that absolute value of the error is bigger in the rectangular case.

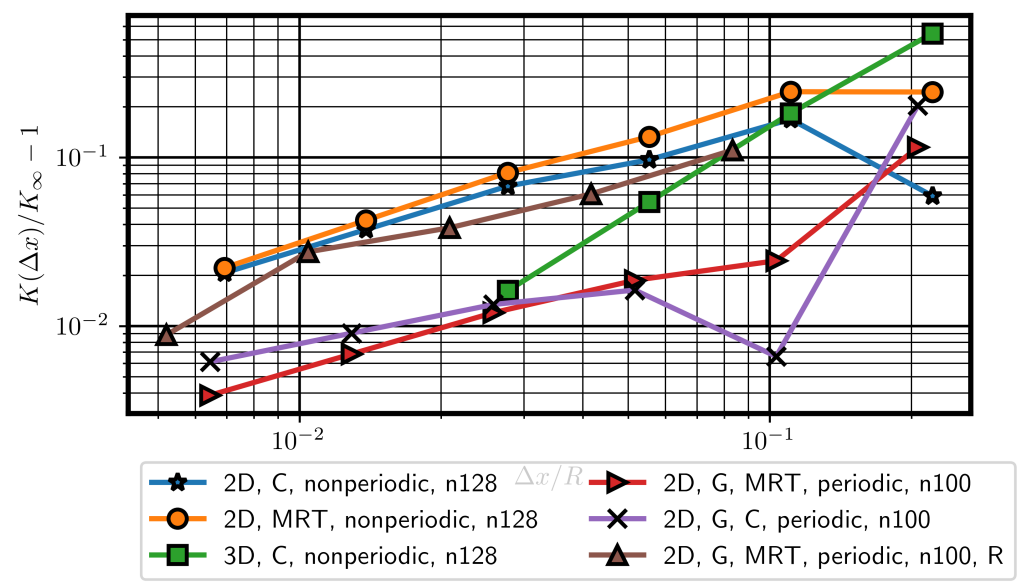

Figure 3: Convergence of the permeability $K$ to extrapolated infinite resolution permeability $K_{\infty}$. Nonperiodic cases refers to setups with symmetry BC combine with pressure BC's used to drive the flow. Periodic cases refers to ones, where mass force was used instead. MRT/C denotes Multiple Relaxation Time and Cumulant LBM collision terms respectively. Letter " $\mathrm{R}$ " denotes single case, where rectangular obstacles where used, instead of circular ones.

Second aspect of the LBM method that we would like to illustrate here is the influence of the numerical roundoff error during density calculations. One could notice that in the low Re LBM simulations, density will be most likely equal to $\rho=1+\epsilon$ which is unfortunate from the 
numerical point of view ( $\epsilon$ could be smaller than $10^{-10}$ ). This source of a round-off error could be reduced, if specialized implementation is used. For example, the introduction of $\tilde{f}_{i}=f_{i}-w_{i}$, where $\rho=1+\sum \tilde{f}_{i}[15]$ significantly reduces those effects. In this study however, the generic LBM code was chosen. Authors expected that influence of the round-off error will be greatly amplified in the case, where the background pressure gradient is introduced using inlet-outlet boundary conditions instead of the gravitational field. Therefore convergence of this worst case are presented in the fig. 3, along with gravitationally driven one. It could be noted that although convergence rate remains on similar level, absolute value of the error is much larger for pressure driven case.

Another parameter that illustrate the compressible character of the LBM method is the standard deviation of the permeability along the flow direction. In the perfectly incompressible regime, it should be zero. Relation between grid resolution and standard deviation of the solution is illustrated in fig. 4, where the standard deviation versus grid resolution was presented for both the gravity and the pressure-jump driven cases. The convergence rate of the standard deviation is similar between cases. For the given value of the grid resolution periodic cases have 2-3 times smaller standard deviation than the pressure driven once. This is because in periodic and gravitationally forced cases the pressure (and therefore density) oscillates around zero, while in the nonperiodic scenario there is linear component in pressure distribution (and therefore in density) which impacts mass conservation. The 3D simulations follow those trends, with standard deviation lying between 2D periodic and non periodic ones. Interestingly, the lowest standard deviation was observed for the case with rectangular obstacles. This is most likely related to the lower area fraction of rectangular obstacles, which leads to lower pressure changes amplitude. It was also found that while an additional error is introduced, it still remain with good agreement with the results obtained by the FEM based solver (see table 1). Additionaly, 3D simulation has a sudden change in convergence ratio (fig. 4); this could be attributed to insufficient resolution in perpendicular direction $z$. In lower resolution cases there where only few nodes per channel height, which might have been insufficient to properly fully represent the $3 \mathrm{D}$ flow field, especially near obstacles.

In conclusion, there is barely no difference in convergence rate of MRT and Cumulant schemes in considered scenarios, both in term of standard deviation and relative error. This was somehow predictable: main difference should lie in the stability for the high Reynolds number and in terms of an order $\mathcal{O}\left(\mathrm{Ma}^{3}\right)$. Most of the presented differences results from the round-off error in collision term implementation, which differ significantly between MRT and Cumulant. Additionally, the convergence and permeability deviation studies indicate that 3D LBM did not reached grid independence fully, the FEM solution would be used as primary in comparisons between 2.5 and 3 dimensional models. 


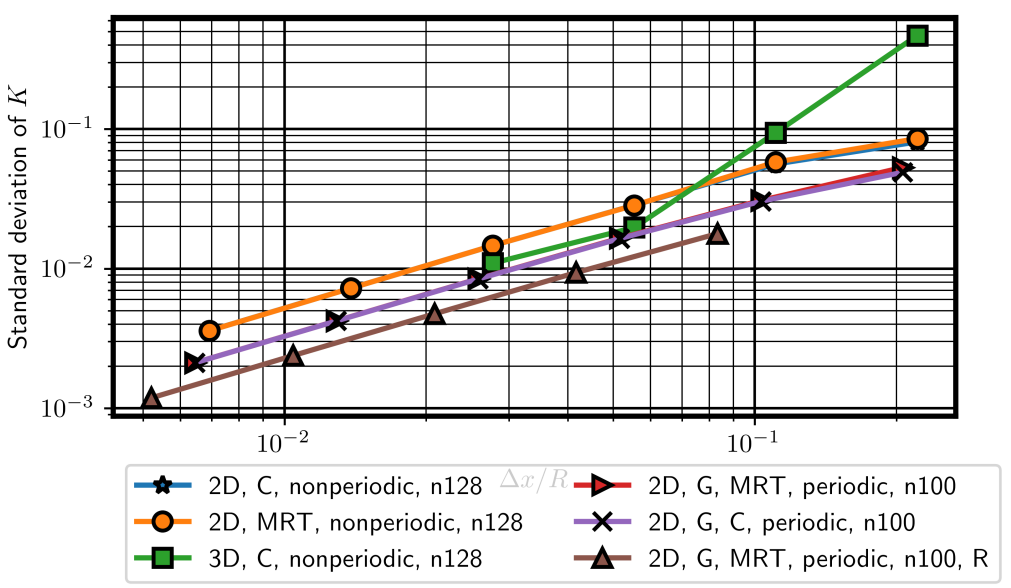

Figure 4: Standard deviation of local permeability (measured in single $y z$ crossection) along the domain in $x$ direction. Nonperiodic cases refers to setups with symmetry BC combine with pressure BC's used to drive he flow. Periodic cases refers to ones, where mass force was used instead. MRT/C denotes Multiple Relaxation Time and Cumulant LBM collision terms. Letter "R" denotes single case, where rectangular obstacles where used, instead of circular ones.

\subsection{2. $M I L A M I N$}

In our FEM simulations, the effective permeability is computed using either line (2D) or surface (3D) integrals of the normal component of the velocity vector. To avoid unnecessary inaccuracies, the integrals are evaluated exactly, using a quadrature rule compliant with the interpolation order of the FEM velocity space. For example, the effective permeability in 3D is evaluated on rectangular $y z$ sections. Each sections is firstly subdivided into a union of nonoverlapping triangular and quadrilateral patches formed by intercepting the section plane with the tetrahedral mesh elements. The quadrilateral patches are further subdivided into triangles. During numerical integration, the velocity is interpolated and sampled in each triangle in a set of points, which are determined according to a suitable exact integration rule for polynomial functions in triangular domains.

Firstly, we study the numerical performance of the 2D and 3D FEM Stokes solvers for periodic and nonperiodic boundary conditions respectively. Fig. 5 (left) presents the convergence of the effective permeability to the extrapolated value of $K_{\infty}$ (obtained by fitting power law in a form of $\left.K_{\infty}+b(\Delta x)^{c}\right)$ as a function of the discretization length $\Delta x / R$ for circular (in 2D and 3D) and square shaped inclusions (in 2D). In the case of circular inclusions, the convergence is quadratic $(c \approx 2)$, and a normalized error of $10^{-3}$ is attained at $\Delta x / R=0.05$. We note that during our convergence tests the net area of the circular inclusions increases with increasing the number of grid points along their rims. For square-shaped inclusions, attaining the same level of error requires $\Delta x / R=0.01$ and a deteriorated, linear convergence $(c \approx 1)$ is observed, which may be related to a poor solution convergence around the sharp inclusion corners. 



Figure 5: A normalized difference between the mean effective permeability and the extrapolated value $K_{\infty}($ left) and a normalized standard deviation of the effective permeability sampled on 100 cross-sections (right) as a function of mesh discretization. 2D FEM simulations for a periodic domain with 100 circular or square inclusions. Hereby rectangular markers refer to rectangular $(\mathrm{R})$ inclusions.

Fig. 5 (right) shows the standard deviations of $K$ calculated in 100 successive sections along $x$-direction and normalized by its average value. The results are presented as a function of discretization length $\Delta x / R$. The normalized standard deviation of $K$ on sections is below $5 \cdot 10^{-3}$ for all cases except one and the standard deviation decay rate is similar for all three curves and it is slightly higher than 2 .

\subsubsection{Computational requirements}

It should be noted here, that this work was not intended to discuss implementation nor hardware issues connected to the LBM and FEM implementations used. For the sake of completeness, hereby we present some details of the performance achieved, but interested reader is directed to performance-dedicated papers.

All of the presented LBM simulations were carried out using CUDA compatible hardware. The biggest 3D LBM simulation had $44 \cdot 10^{6}$ lattice points, and 27 -neigbours lattice nodes were used which required $21.4 \mathrm{~GB}$ of memory. The average iteration speed was $650 \cdot 10^{6}$ lattice points updates per second on two nVidia Tesla K40d accelerators on single cluster node. There were no convergence criteria implemented, and simulations were stopped after predefined number of time steps. It took $7.6 h$ to complete $\sim 400 k$ time steps of the mentioned $3 \mathrm{D}$ simulation. For $2 \mathrm{D} / 2.5 \mathrm{D}$ cases, iteration speed was up to $10^{7}$ lattice points updates per second for large enough cases. Largest meshes in $2 \mathrm{D} / 2.5 \mathrm{D}$ had $\sim 17 \cdot 10^{6}$ lattice points and $2.7 \mathrm{~Gb}$ of memory was used. On a single K40d it took less then 2 hours to evaluate $\sim 400 k$ time steps. There was no noticeable difference between $2 \mathrm{D}$ and $2.5 \mathrm{D}$ cases, because same code was used in both cases.

In the 3D FEM simulations the largest had $16 \cdot 10^{6}$ nodes (around $48 \cdot 10^{6}$ velocity degrees of freedom) and such problem occupied around 128GB of RAM. We had used dual socket server with Intel CPU E5-2650 v2 processors and 128GB of DDR3 RAM (1866MHz). To resolve 3D velocity field, it took around $4 h$ to solve single case. Single $2 / 2.5 \mathrm{D}$ FEM case with $10^{6}$ nodes occupies around $2 \mathrm{~GB}$ of memory and takes around 20 seconds to solve. 


\section{The 2.5D Stokes-Brinkman model}

\subsection{The Stokes-Brinkman approach for fracture flow}

The Stokes-Brinkman, or the Brinkman, model is traditionally used for combing fracture (Stokes) and porous (Darcy) flow in dual porosity media [95, 96, 97, 98, 99]. In our case, the Stokes-Brinkman model arises by integrating the flow equations in the direction perpendicular to the main fracture plane. Such approach is present from over a century, especially in context of analytical studies single obstacle (i.e. [100]), or more recent in numerical analyses (i.e. [73, 64]). Averaged equations would play the role of an upscaled 2.5D model of fracture flow.

We assume that fluid motion is governed by Stokes equation:

$$
\begin{cases}\mu \sum_{k} \frac{\partial^{2}}{\partial x_{k}^{2}} u_{i}-\frac{\partial}{\partial x_{i}} p & =F_{i} \\ \sum_{i} \frac{\partial}{\partial x_{i}} u_{i}= & 0\end{cases}
$$

where $u_{i}$ and $p$ denote the velocity components and pressure, respectively. The driving mass force is denoted by $F_{i}$. Assuming that velocity components are parabolic in $z$ direction (perpendicular to the fracture plane) Stokes equations could be integrated in $z$ direction to obtain a reduced model:

$$
\left\{\begin{array}{l}
\mu\left[\left(\frac{\partial^{2}}{\partial^{2} x}+\frac{\partial^{2}}{\partial^{2} y}\right)\left\langle u_{x}\right\rangle\right]-\frac{12 \mu}{h^{2}}\left\langle u_{x}\right\rangle-\frac{\partial}{\partial x}\langle p\rangle=\left\langle F_{x}\right\rangle \\
\mu\left[\left(\frac{\partial^{2}}{\partial^{2} x}+\frac{\partial^{2}}{\partial^{2} y}\right)\left\langle u_{y}\right\rangle\right]-\frac{12 \mu}{h^{2}}\left\langle u_{y}\right\rangle-\frac{\partial}{\partial y}\langle p\rangle=\left\langle F_{y}\right\rangle
\end{array}\right.
$$

where constant gap height is denoted by $h$, and $u_{z}$ was dropped. Symbol \langle\rangle denotes depthaveraged values $\left(h\langle\bullet\rangle=\int_{0}^{h} \bullet d z\right)$. After integrating along $z$, the continuity equation is

$$
\frac{\partial}{\partial x}\left\langle u_{x}\right\rangle+\frac{\partial}{\partial y}\left\langle u_{y}\right\rangle+\left.u_{z}\right|_{0} ^{h}=0
$$

where the last term could be dropped. This finally leads to a $2 \mathrm{D}$ system

$$
\begin{cases}\mu \nabla^{2}\langle\mathbf{u}\rangle & =\langle\mathbf{F}\rangle-\frac{12 \mu}{h^{2}}\langle\mathbf{u}\rangle-\nabla\langle p\rangle \\ \nabla \cdot\langle\mathbf{u}\rangle & =0\end{cases}
$$

where $\langle\mathbf{u}\rangle=\left[\left\langle u_{x}\right\rangle,\left\langle u_{y}\right\rangle\right]$. In this study we use $\frac{12 \mu}{h^{2}}\langle\mathbf{u}\rangle$ as the Brinkman (mass force) term in momentum equation to model finite gap height and to obtain 2.5D approximation of 3D solution. It should be noted that despite the fact that $z$ velocity component have has a zero-average, it could influence permeability of the medium.

\subsection{Implementation of the Stokes-Brinkman 2.5D model}

In case of the LBM solution, where EDM force term was used, physical fluid velocity is obtained with

$$
\rho \mathbf{u}=\sum \mathbf{e}_{j} f_{j}-\mathbf{F} \frac{\Delta t}{2}
$$

On the other hand, in 2.5D Brinkman model, flow resistance due to finite aperture is represented by force term proportional to velocity

$$
\mathbf{F}_{B}=\frac{12 \mu}{b^{2}} \mathbf{u}
$$

where $b$ is the local aperture. Combining (9) and (8) we obtain

$$
\rho \mathbf{u}=\sum \mathbf{e}_{j} f_{j}-K \rho \mathbf{u}
$$


where

$$
K=\frac{\Delta t}{2} \frac{12 \nu}{b^{2}}
$$

therefore fluid velocity is equal to

$$
\mathbf{u}=\frac{\sum \mathbf{e}_{j} f_{j}}{\rho(1+K)}
$$

It should be noted that as collision does not change momentum, so the force during collision could be evaluated as

$$
\mathbf{F}=\mathbf{G}+K \frac{\sum \mathbf{e}_{j} f_{j}}{\rho(1+K)}
$$

where $\mathbf{G}$ is the gravitational acceleration term. Those results are generic enough to be directly implemented in the Cumulant collision operator.

Similarly, in the FEM solution, the drag force term arising in the 2.5D Stokes-Brinkman model can be incorporated into the code by adding a mass matrix term to the velocity stiffness matrix A (see Section 1.2.2).

\subsection{Validation of the Stokes-Brinkman LBM and FEM implementations against an analytical solution of Spielman and Goren (1968)}

Spielman and Goren [101] first derived an analytical solution to the Stokes-Brinkman equation for the flow around an isolated circular cylinder in terms of the modified Bessel functions. A similar analytical solution was further used in other works [99, 102, 103].

In our validation tests, we choose a fracture aperture equal to 0.566 of the inclusion radius $(H / R=0.5666$, same as in a multi-obstacle problem chosen in the later analysis, where area fraction was equal to 0.45) and set the permeability term in the Stokes-Brinkman equation accordingly. We use both TCLB and MILAMIN Stokes-Brinkman implementations to obtain numerical solutions for validation purposes. The ratio between the obstacle radius and numerical domain length was chosen as $L / R=20$. Single FEM run was also performed for $L / R=2 \cdot 10^{5}$. FEM simulations used a nonuniform mesh significantly strongly refined near the obstacle. The tests were run using periodic boundary conditions and gravitational forcing.

Both methods were tested for convergence based on the effective permeability $K_{\infty}$ (fig. 6). LBM results converge with a rate of 1.1 and the effective permeability measured in FEM simulation exhibits a quadratic convergence. Velocity profiles were also evaluated and validated along three cross sections (fig 6 ): $x$ velocity component profile is presented along $x$ axis (from domain edge to stagnation point at the obstacle) and along $y$ axis (from domain edge to the obstacle edge). The $y$ component of the velocity vector was plot on an off-central line parallel to the $x$ axis. In the case of $L / R=20$ a slight departure from the reference analytical solution is observed, especially near the domain boundary. The effect is due to the influence of the finite domain size, which is not visible, if FEM solution for $L / R=2 \cdot 10^{5}$ is considered. This indicates, that departure of the velocity field in the high resolution LBM solution presented in fig. 6 is related to finite domain size rather than to insufficient geometrical representation of the obstacle geometry. Unfortunately, much bigger domain is impractical for LBM simulations without grid resolution variable in space. There is no evident difference between LBM and FEM results for $L / R=20$, so we expect that LBM results also converge to the analytical solution for a sufficiently large domain. It should be also noted, that relative permeability error is far below $1 \%$ (see fig. 6) for low resolution LBM and FEM simulations. The high resolution simulations yielded $\sim 10^{-4}$ accuracy for LBM and $\sim 10^{-7}$ in FEM. 

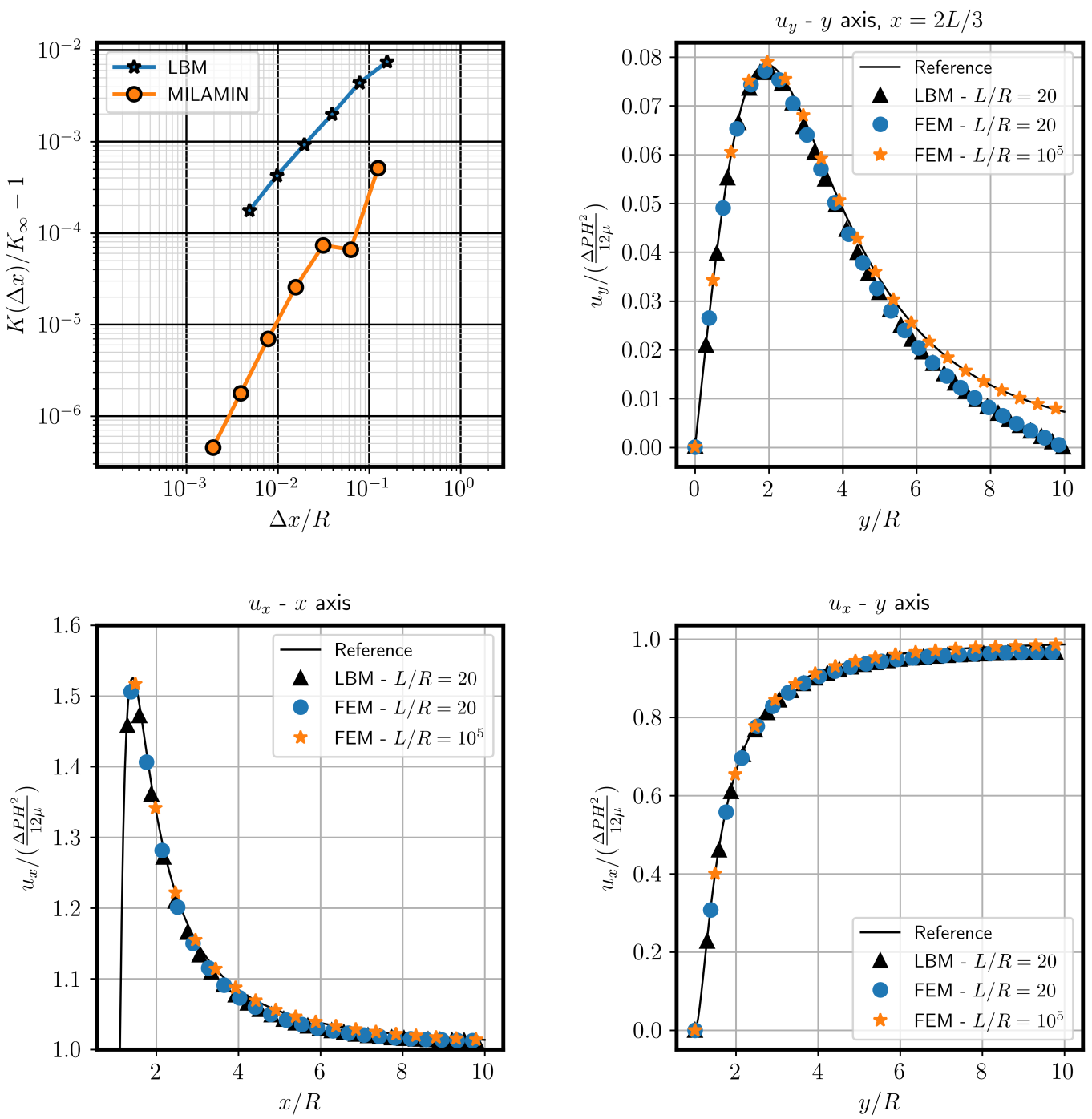

Figure 6: Convergence of the single obstacle permeability to infinite resolution solution $K_{\infty}$ (upper-left) and velocity profile around the obstacle compared with the analytical solution for an infinite domain ("Reference", by Spielman and Goren [101]) in three cross-sections. Coordinate system origin is placed at the obstacle center. The $y$ component of the velocity vector was plot on an off-central line parallel to the $x$ axis for $x / L=2 / 3$ (top-right). The $x$ velocity component profile is presented along $x$ axis (from domain edge to stagnation point at the obstacle) and along $y$ axis (from domain edge to the obstacle edge), bottom left and right figures respectively. Circular markers denotes truncated domain with $L / R=20$, while stars were used to present large domain FEM solution.

\subsection{Evaluation of the 2.5D model vs. full 3D solution}

We test the convergence and accuracy of the 2.5D Stokes-Brinkman model using both the LBM and FEM based implementations for systems with multiple obstacles. High-resolution $3 \mathrm{D}$ simulations were run to obtain the reference solution to the original Stokes problem. The fracture aperture is set to $H / R=0.5666$ (area fraction was equal to 0.45), free slip boundary conditions are imposed at the $y$ walls, and the flow is driven by a pressure difference applied in 
the $\mathrm{x}$ direction.

The numerical convergence of the effective permeability towards the extrapolated value obtained in the case of $2.5 \mathrm{D}$ LBM simulations $K_{\infty}$ (fig. 7) is slower than in the case of $3 \mathrm{D}$ simulations. A similar effect was observed in $2 \mathrm{D}$ simulations. The relative difference between the 2.5D and 3D permeability obtained using the LBM solver was around 10\% (see summary in table 2 ), with $K_{\infty}=2.23 \cdot 10^{-3}$ for the 3D LBM case. FEM based $2.5 \mathrm{D}$ and 3D models converge with same rate. The relative difference between the extrapolated fracture permeabilities $K_{\infty}$ in 2.5D and 3D FEM solutions is less than $8 \%$, with $K_{\infty}=2.31 \cdot 10^{-3}$ in the case of $3 \mathrm{D}$ simulations.

In fig. 8 the standard deviation of permeability measured for various $x$ sections along the domain is presented for both FEM and LBM solutions. In both LBM and FEM simulations, permeability obtained in $2.5 \mathrm{D}$ simulation exhibit a standard deviation similar to the results obtained in 3D simulations. The only exception is the lowest resolution 3D LBM case. Finally, table 2 summarized the obtained data for all analyzed cases and methods.
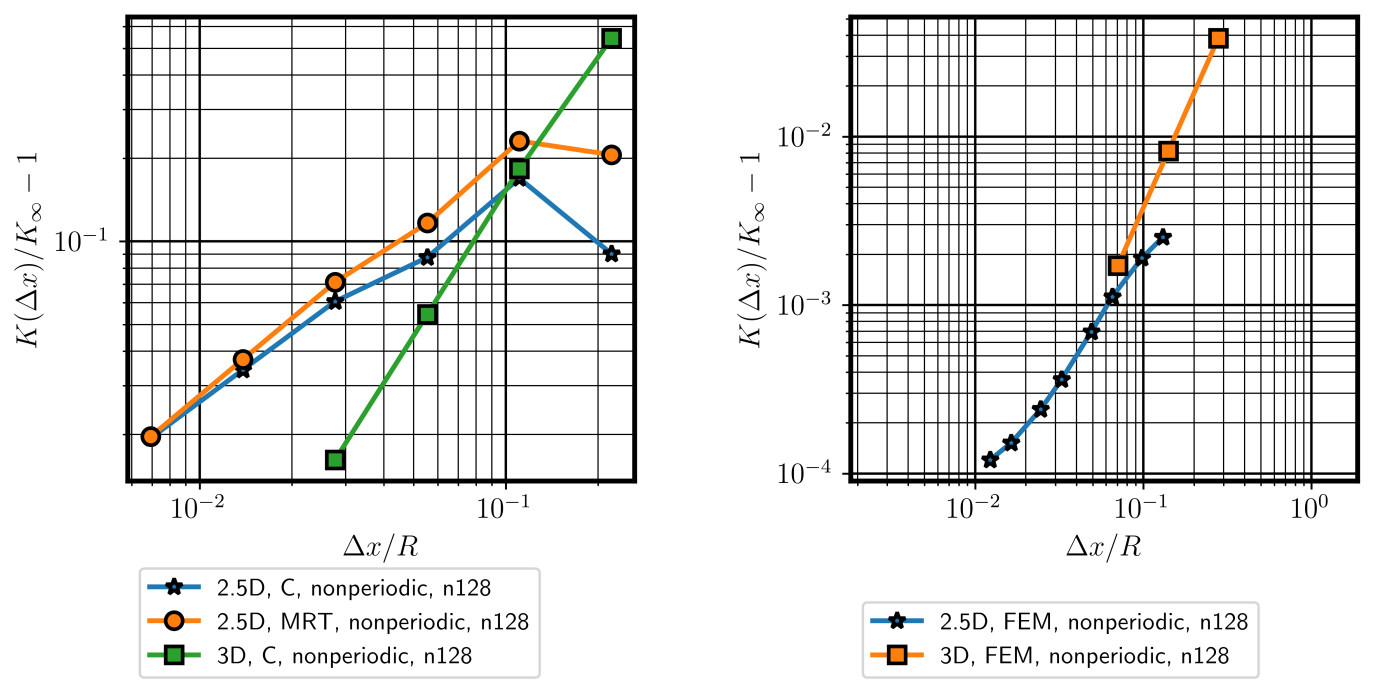

Figure 7: Convergence of the 2.5D and 3D permeability to extrapolated one. The highest resolution solution of the 3D LBM used $\sim 23 \cdot 10^{6}$ points, while 3 D FEM mesh consist of $\sim 16 \cdot 10^{6}$ points and $\sim 3.3 \cdot 10^{6}$ elements. Sudden drop in LBM 2.5D solutions (left figure) for low resolution simulations is a result of insufficient discretisation which lead to larger error. In this case those data points give seemingly better accuracy in term of permeability, but mesh independence study reveal that at least 50 nodes per radius are necessary to recover proper solution. On the other hand, 3D case seems to give higher convergence rate and smaller relative error in $3 \mathrm{D}$ case, but again the result might be influence by relatively low resolution of $3 \mathrm{D}$ LBM case. 

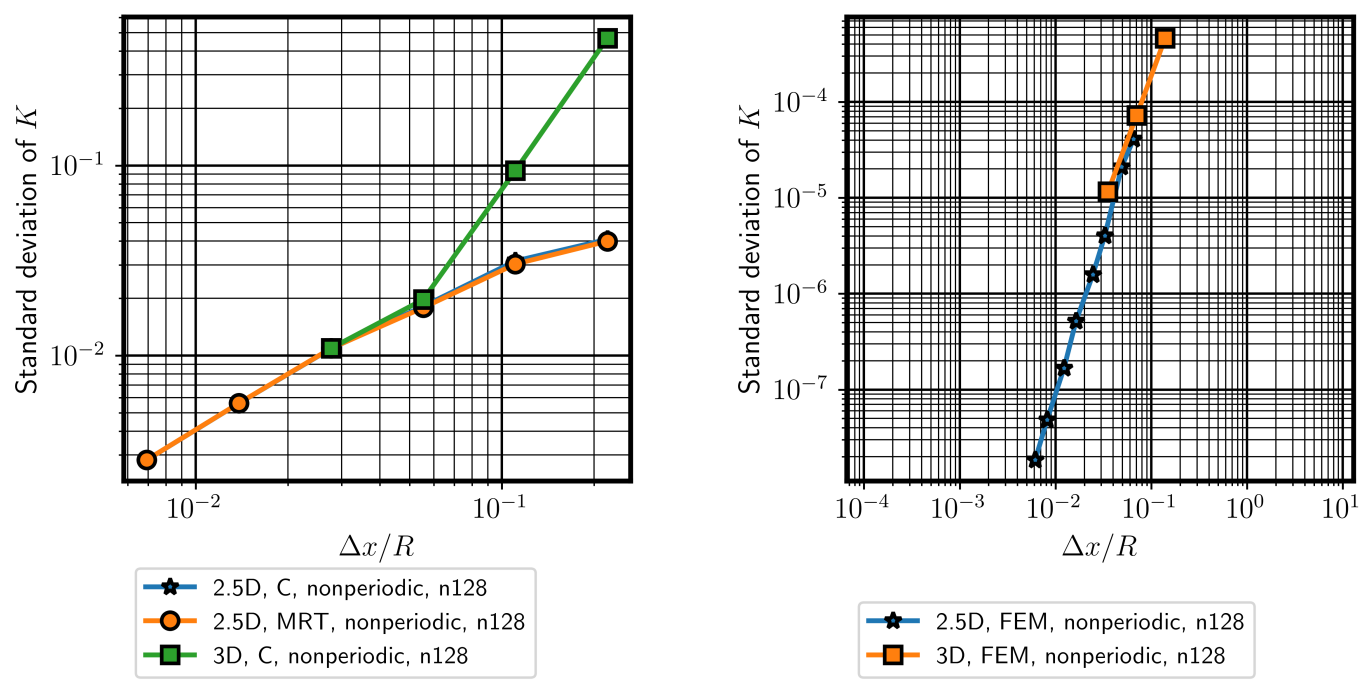

Figure 8: Convergence of the standard deviation of permeability along $x$ axis. Presented convergence character is almost identical to pure $2 \mathrm{D}$ solution in LBM, and fallows $3 \mathrm{D}$ in FEM case.

\begin{tabular}{|c|c|c|c|c|c|c|c|c|}
\hline & Name & \# of. incl. & frac. & $H / R$ & $\dot{\vec{\Phi}}$ & forcing & $L / R$ & $K_{\infty} / R^{2} \cdot 10^{3}$ \\
\hline \multicolumn{9}{|c|}{ Periodic domain, $2.5 \mathrm{D}$ model } \\
\hline 4 & 2.5D, G, C, periodic, n100 & \multirow{2}{*}{100} & \multirow{2}{*}{0.45} & \multirow{2}{*}{1.058} & \multirow{2}{*}{$\mathrm{Y}$} & \multirow{2}{*}{$G$} & \multirow{2}{*}{26.46} & 10.028 \\
\hline 4 & 2.5D, G, FEM, periodic, n100 & & & & & & & 10.017 \\
\hline \multicolumn{9}{|c|}{ Non-periodic domain, 2.5D and 3D model } \\
\hline \multirow{3}{*}{5} & 25D, MRT, nonperiodic, n128 & \multirow{5}{*}{128} & \multirow{5}{*}{0.5} & \multirow{5}{*}{0.567} & \multirow{5}{*}{$\mathrm{N}$} & \multirow{5}{*}{$\Delta P$} & \multirow{5}{*}{28.33} & 2.42 \\
\hline & 25D, C, nonperiodic, n128 & & & & & & & 2.496 \\
\hline & 25D, FEM, nonperiodic, n128 & & & & & & & 2.483 \\
\hline \multirow{2}{*}{6} & 3D, C, nonperiodic, n128 & & & & & & & 2.15 \\
\hline & 3D, FEM, nonperiodic, n128 & & & & & & & 2.31 \\
\hline
\end{tabular}

Table 2: Solutions summary of the accuracy tests of the 2.5D model. "C" refers to solutions obtained using Cumulant LBM variant, "MRT" denotes Multiple Relaxation Time variant of LBM, see 1.2.1. "FEM" refers to solution obtained using finite element approach. "G" are cases driven by gravity acceleration.

Figures 10 and 9 illustrate the local differences between 2.5D and fully resolved 3D solution, for both FEM and LBM based solvers. In fig. 9 details of of $z$ depth-averaged $x$ component of the velocity is presented along $y$ crossection of the 2D domain (through the midpoint of the domain). Higher discrepancies are present at domain boundaries in the $y$ direction. The discrepancy is higher between 3D and 2.5D models than between the two solution methods (FEM vs LBM). This indicates that the difference is related to the $2.5 \mathrm{D}$ approximation rather than the numerical accuracy. The derived Stokes-Brinkman model builds on an assumption that the velocity components are parabolic in $z$. We introduce $u_{i}^{2 D}$ as a scalar product of the velocity components obtained from 3D simulations and a parabola

$$
u_{i}^{2 D}=\frac{1}{b} \int_{0}^{b} u_{i}^{3 D} f(z) d z
$$


where $u_{i}^{3 D}$ are the velocity components of $3 \mathrm{D}$ flow field. The function $f(z)$ is a defined as $f(z)=a z^{2}+b z+c$, with $a, b, c$ such that

$$
\int_{0}^{b}[f(z)]^{2} d z=1
$$

The difference between $u_{i}^{2 D}$ and $z$-averages $3 \mathrm{D}$ solution measures the non-parabolic part of the 3D flow field. Departure from parabolic profile measured with such criterion is illustrated in fig. 10, where histogram of the difference for high-resolution 3D case is presented. The non-parabolic component does not vanish with increasing numerical resolution. This is an expected result, if we consider an analytical solution for a single cylindrical obstacle, which shows a non-zero $z$ component of the velocity in the vicinity of the obstacle and a non-strictly parabolic velocity field [104]. The second leading order of pressure field is strictly connected to antisymmetric part of $z$ component of the velocity field. This part of the solution is dropped in the Brinkman approximation, leading to the second-order discrepancy between the two. The gradient of this higher order component of the pressure field directly influences the parabolic component of the solution, therefore it is also the main reason why $3 \mathrm{D}$ and $2.5 \mathrm{D}$ solutions do not converge to the same $K_{\infty}$. Interestingly, LBM solution show pike in low-velocity-error part of the $x$ velocity histogram (fig. 10), which is not present in FEM solution. Closer observation reveals, that similar, but smaller, disturbance exists in $y$ profile. The FEM solution does not have such feature, nor for the $y$ nor $x$ velocity error. This might indicate some additional futures of the 3D LBM velocity field, that are not present in FEM solution. One of such element could be the present of non-linear part of the momentum equation. It should be recalled here, that LBM solve Navier-Stokes equation, while FEM was a Stokes equation solver. Despite small/vanishing Reynolds number studies presented here, those additional terms may lead to different character of the projection onto parabolic velocity field.
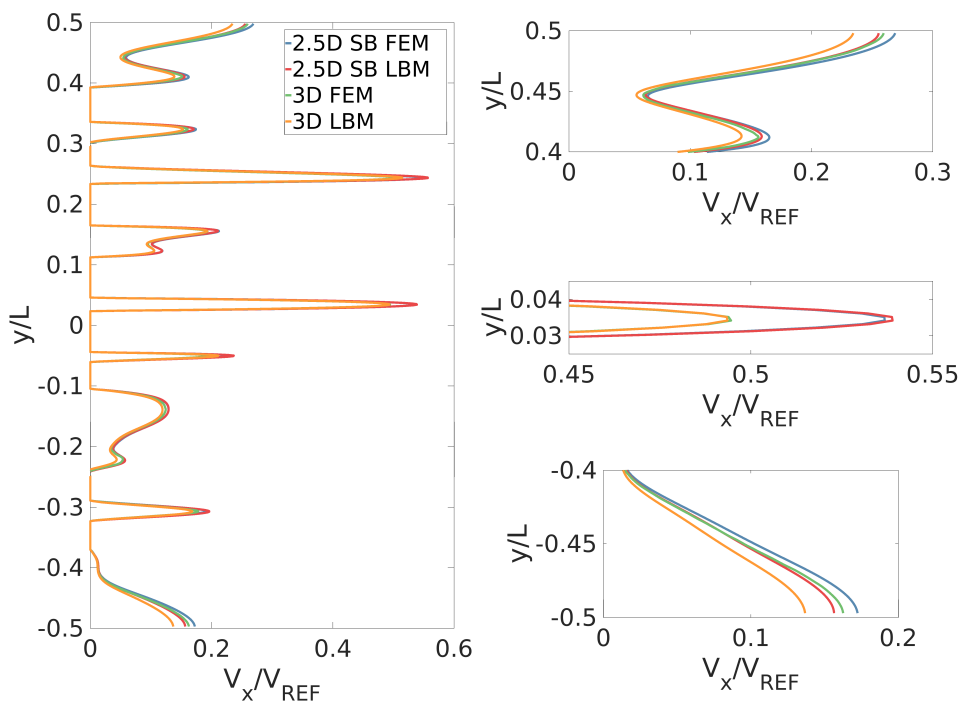

Figure 9: $V_{x}$ profiles along line $\mathrm{y}$ (for $\mathrm{x}=0$ and $\mathrm{z}=0$ for $3 \mathrm{D}$ case) for 128 proppant grains and nonperiodic boundary conditions. $V_{R E F}$ is the velocity in an empty domain (without proppants). Due to the very small difference between the profile lines, the interested reader is referred to the online version of this work.. The differences are highlighted on the right-hand side zooms (2.5D FEM Stokes-Brinkmann - blue, 2.5D LBM Stokes-Brinkmann red, 3D FEM - green, 3D LBM - orange). 

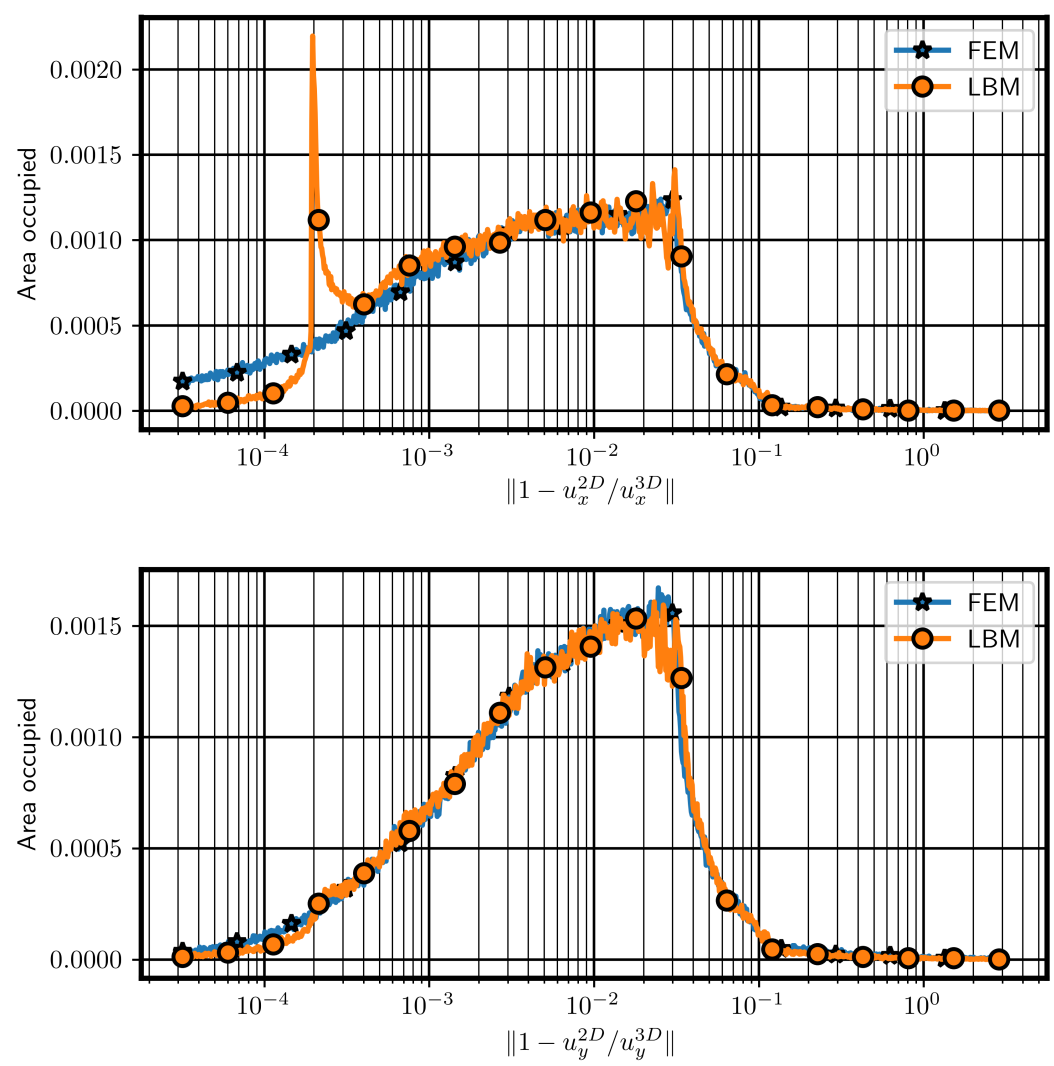

Figure 10: Histogram of the relative difference between averaged fully 3D velocity field and parabolic in $z$ profile assumed in Brinkman approximation. Upper figure presents $x$ component, lower one presents $y$ component. Both LBM and FEM data were taken from highest available resolution. On both figures FEM and LBM lines almost overlap. In the upper picture distinguish peak belong to LBM line. It is present (but vanishing) for all resolutions available, but with reduced amplitude and for lower relative difference value.

\section{Conclusions}

We have studied numerical performance and accuracy of MRT and Cumulant LBM schemes for Stokes and Stokes-Brinkman fracture flow models. FEM models and an analytical solution to the Stokes-Brinkman flow around an isolated cylinder have been used to provide an independent validation. The differences between the computed fracture permeabilities are below $1 \%$ in the case of $2 \mathrm{~d}$ fracture models with multiple obstacles. The observed discrepancy can be partly related to numerical compressibility and inaccurate representation of circular obstacles inherent to LBM models on structured computational meshes. The mismatch is less pronounced (below $0.3 \%$ ) in the case of rectangular obstacles and gravity loading. The relative difference between the LBM and FEM results using the 2.5D Stokes-Brinkman model is below $1 \%$. An almost perfect agreement has been observed between the numerical solutions and the analytical solution for the Stokes-Brinkman flow around an isolated, circular obstacles (some discrepancies have been introduced due to finite sized domain). The difference between the FEM-based 3D and the corresponding $2.5 \mathrm{D}$ results is around $6 \%$, and around $10 \%$ in the case of LBM simulations. Although, the in-plane resolution used in the 2D studies have not been achieved in the 3D case, 
we related the observed discrepancies to the approximate nature of the 2.5D Stokes-Brinkman model. In conclusion:

- Both FEM and LBM are able to properly resolve two- and three-dimensional Stokes models with a similar level of accuracy. It is possible to introduce a Brinkman term into the two methods and obtain a similar level of accuracy. Both the LBM and FEM-based StokesBrinkman models are capable of accurately reproducing the analytical solution available for an isolated single obstacle.

- High resolution $(\Delta x / R=100)$ meshes might me necessary to reduce the effective permeability error below $1 \%$ in LBM simulations. A higher accuracy could be achieved with a dedicated, incompressible LBM scheme together with a higher order BCs method. On the other hand, the FEM solution shows a slower convergence for rectangular obstacles, which could be attributed to a poor convergence around the sharp inclusion corners.

- We estimate the error introduced by the $2.5 \mathrm{D}$ approximation at around at least $6 \%$.

- The velocity perturbations that are introduced around the obstacles are not fully captured by the parabolic velocity profile inherent to the $2.5 \mathrm{D}$ Stokes-Brinkman model. It could be beneficial to develop and test higher order 2.5D approximations to single-phase flow in fractures with obstacles.

\section{Acknowledgment}

Authors acknowledge funding in the framework of the ShaleSeq project (Physicochemical effects of $\mathrm{CO} 2$ sequestration in the Pomeranian gas bearing shales) co-funded from Norway Grants in the Polish-Norwegian Research Programme operated by the National Centre for Research and Development, grant no. POL-NOR/234198/100/2014.. This research was also supported in by PL-Grid Infrastructure. The GPGPU calculations were performed on Prometheus HPC unit at the Academic Computer Centre CYFRONET in Cracow. The FEM calculations were performed using the Computational Geology Laboratory (PGI-NRI) group computer resources.

\section{Bibliography}

[1] G. E. King, et al., Thirty years of gas shale fracturing: what have we learned?, in: SPE Annual Technical Conference and Exhibition, Society of Petroleum Engineers, 2010.

[2] B. Legarth, E. Huenges, G. Zimmermann, Hydraulic fracturing in a sedimentary geothermal reservoir: Results and implications, International Journal of Rock Mechanics and Mining Sciences 42 (7) (2005) 1028-1041.

[3] A. Genter, K. Evans, N. Cuenot, D. Fritsch, B. Sanjuan, Contribution of the exploration of deep crystalline fractured reservoir of Soultz to the knowledge of enhanced geothermal systems (EGS), Comptes Rendus Geoscience 342 (7-8) (2010) 502-516. doi:10.1016/j.crte.2010.01.006.

[4] A. Khanna, A. Kotousov, J. Sobey, P. Weller, Conductivity of narrow fractures filled with a proppant monolayer, Journal of Petroleum Science and Engineering 100 (2012) 9-13.

[5] J. Zhang, L. Ouyang, D. Zhu, A. Hill, Experimental and numerical studies of reduced fracture conductivity due to proppant embedment in the shale reservoir, Journal of Petroleum Science and Engineering 130 (2015) 37-45. 
[6] H. D. Brannon, M. R. Malone, A. R. Rickards, W. D. Wood, J. R. Edgeman, J. L. Bryant, et al., Maximizing fracture conductivity with proppant partial monolayers: theoretical curiosity or highly productive reality?, in: SPE Annual Technical Conference and Exhibition, Society of Petroleum Engineers, 2004.

[7] R. Hale, R. Ranjan, C. Hidrovo, Capillary flow through rectangular micropillar arrays, International Journal of Heat and Mass Transfer 75 (2014) 710-717.

[8] Z. Chai, B. Shi, A novel lattice Boltzmann model for the Poisson equation, Applied Mathematical Modelling 32 (10) (2008) 2050-2058. doi:10.1016/j.apm.2007.06.033.

[9] M. Wang, J. Wang, S. Chen, N. Pan, Electrokinetic pumping effects of charged porous media in microchannels using the lattice Poisson-Boltzmann method, Journal of Colloid and Interface Science 304 (1) (2006) 246-253. doi:10.1016/j.jcis.2006.08.050.

[10] X. Yang, B. Shi, Z. Chai, Z. Guo, A Coupled Lattice Boltzmann Method to Solve Nernst-Planck Model for Simulating Electro-osmotic Flows, Journal of Scientific Computing 61 (1) (2014) 222-238. doi:10.1007/s10915-014-9820-6.

[11] M. Geier, A. Fakhari, T. Lee, Conservative phase-field lattice Boltzmann model for interface tracking equation, Physical Review E 91 (6). doi:10.1103/PhysRevE.91.063309.

[12] P. J. Dellar, Incompressible limits of lattice Boltzmann equations using multiple relaxation times, Journal of Computational Physics 190 (2) (2003) 351-370.

[13] X. He, L.-S. Luo, Lattice Boltzmann Model for the Incompressible Navier-Stokes Equation, Journal of Statistical Physics 88 (3/4) (1997) 927-944. doi:10.1023/B:JOSS.0000015179.12689.e4.

[14] L. Zhang, S. Yang, Z. Zeng, L. Yin, Y. Zhao, J. W. Chew, Consistent lattice Boltzmann methods for incompressible axisymmetric flows, Physical Review E 94 (2) (2016) 023302. doi:10.1103/PhysRevE.94.023302.

[15] P. A. Skordos, Initial and boundary conditions for the lattice Boltzmann method, Physical Review E 48 (6) (1993) 4823-4842. doi:10.1103/PhysRevE.48.4823.

[16] M. K. Misztal, A. Hernandez-Garcia, R. Matin, H. O. Sørensen, J. Mathiesen, Detailed analysis of the lattice Boltzmann method on unstructured grids, Journal of Computational Physics 297 (2015) 316-339. doi:10.1016/j.jcp.2015.05.019.

[17] G. R. McNamara, G. Zanetti, Use of the Boltzmann Equation to Simulate LatticeGas Automata, Physical Review Letters 61 (20) (1988) 2332-2335. doi:10.1103/PhysRevLett.61.2332.

[18] H. Chen, S. Chen, W. H. Matthaeus, Recovery of the Navier-Stokes equations using a lattice-gas Boltzmann method, Physical Review A 45 (8) (1992) R5339-R5342. doi:10.1103/PhysRevA.45.R5339.

[19] S. Chen, H. Chen, D. Martnez, W. Matthaeus, Lattice Boltzmann model for simulation of magnetohydrodynamics, Physical Review Letters 67 (27) (1991) 3776-3779. doi:10.1103/PhysRevLett.67.3776.

[20] J. Hardy, Y. Pomeau, O. de Pazzis, Time Evolution of a Two-Dimensional Classical Lattice System, Physical Review Letters 31 (5) (1973) 276-279. doi:10.1103/PhysRevLett.31.276. 
[21] D. Rothman, Cellular-automaton fluids: A model for flow in porous media, GEOPHYSICS 53 (4) (1988) 509-518. doi:10.1190/1.1442482.

[22] D. d'Humières, P. Lallemand, U. Frisch, Lattice Gas Models for 3d Hydrodynamics, EPL (Europhysics Letters) 2 (4) (1986) 291. doi:10.1209/0295-5075/2/4/006.

[23] Y. H. Qian, D. D'Humières, P. Lallemand, Lattice BGK Models for Navier-Stokes Equation, EPL (Europhysics Letters) 17 (6) (1992) 479. doi:10.1209/0295-5075/17/6/001.

[24] S. Succi, R. Benzi, M. Vergassola, A. Cancelliere, Hydrodynamic behaviour of the Lattice Boltzmann Equation, in: Numerical Methods for the Simulation of Multi-Phase and Complex Flow, Springer, 1990, pp. 39-48.

[25] B. Ferréol, D. H. Rothman, Lattice-Boltzmann simulations of flow through Fontainebleau sandstone, Transport in Porous Media 20 (1-2) (1995) 3-20. doi:10.1007/BF00616923.

[26] H. Andrä, N. Combaret, J. Dvorkin, E. Glatt, J. Han, M. Kabel, Y. Keehm, F. Krzikalla, M. Lee, C. Madonna, M. Marsh, T. Mukerji, E. H. Saenger, R. Sain, N. Saxena, S. Ricker, A. Wiegmann, X. Zhan, Digital rock physics benchmarks - part II: Computing effective properties, Computers \& Geosciences 50 (2013) 33-43. doi:10.1016/j.cageo.2012.09.008.

[27] S. Succi, The Lattice Boltzmann Equation for Fluid Dynamics and Beyond, Oxford University Press, USA, 2001.

[28] W. Regulski, J. Szumbarski, Ł. Łaniewski-Wołłk, K. Gumowski, J. Skibiński, M. Wichrowski, T. Wejrzanowski, Pressure drop in flow across ceramic foams-A numerical and experimental study, Chemical Engineering Science 137 (2015) 320-337. doi:10.1016/j.ces.2015.06.043.

[29] H. Cho, N. Jeong, H. J. Sung, Permeability of microscale fibrous porous media using the lattice Boltzmann method, International Journal of Heat and Fluid Flow 44 (2013) 435443. doi:10.1016/j.ijheatfluidflow.2013.07.013.

[30] Y. Ning, S. He, T. Chen, Y. Jiang, G. Qin, Simulation of Shale Gas Transport in 3d Complex Nanoscale-Pore Structures Using the Lattice Boltzmann Method, Society of Petroleum Engineers, 2015. doi:10.2118/176997-MS.

[31] L. Chen, L. Zhang, Q. Kang, H. S. Viswanathan, J. Yao, W. Tao, Nanoscale simulation of shale transport properties using the lattice Boltzmann method: permeability and diffusivity, Scientific Reports 5 (2015) 8089. doi:10.1038/srep08089.

[32] E. S. Boek, M. Venturoli, Lattice-Boltzmann studies of fluid flow in porous media with realistic rock geometries, Computers \& Mathematics with Applications 59 (7) (2010) 23052314. doi:10.1016/j.camwa.2009.08.063.

[33] J. A. White, R. I. Borja, J. T. Fredrich, Calculating the effective permeability of sandstone with multiscale lattice Boltzmann/finite element simulations, Acta Geotechnica 1 (4) (2006) 195-209. doi:10.1007/s11440-006-0018-4.

[34] F. Brezzi, M. Fortin, Mixed and Hybrid Finite Element Methods, Springer-Verlag New York, Inc., New York, NY, USA, 1991.

[35] H. Elman, D. Silvester, A. Wathen, Finite Elements and Fast Iterative Solvers : with Applications in Incompressible Fluid Dynamics: with Applications in Incompressible Fluid Dynamics, Numerical Mathematics and Scientific Computation, OUP Oxford, 2005. 
[36] M. Adamuszek, M. Dabrowski, D. W. Schmid, Folder: A numerical tool to simulate the development of structures in layered media, Journal of Structural Geology 84 (2016) 85101.

[37] B. J. P. Kaus, S. M. Schmalholz, 3d finite amplitude folding: Implications for stress evolution during crustal and lithospheric deformation, Geophysical Research Letters 33 (14) (2006) n/a-n/a, 114309. doi:10.1029/2006GL026341.

[38] D. W. Schmid, M. Dabrowski, M. Krotkiewski, Evolution of large amplitude 3D fold patterns: A FEM study, Physics of the Earth and Planetary Interiors 171 (2008) 400-408. doi:10.1016/j.pepi.2008.08.007.

[39] G. Stadler, M. Gurnis, C. Burstedde, L. C. Wilcox, L. Alisic, O. Ghattas, The dynamics of plate tectonics and mantle flow: From local to global scales, science 329 (5995) (2010) 1033-1038.

[40] E. Mulyukova, B. Steinberger, M. Dabrowski, S. V. Sobolev, Survival of llsvps for billions of years in a vigorously convecting mantle: Replenishment and destruction of chemical anomaly, Journal of Geophysical Research: Solid Earth 120 (5) (2015) 3824-3847, 2014JB011688. doi:10.1002/2014JB011688.

[41] M. Dabrowski, D. W. Schmid, Y. Y. Podladchikov, A two-phase composite in simple shear: Effective mechanical anisotropy development and localization potential, Journal of Geophysical Research: Solid Earth 117 (B8) (2012) n/a-n/a, b08406. doi:10.1029/2012JB009183.

[42] N. S. Mancktelow, Finite-element modelling of shear zone development in viscoelastic materials and its implications for localisation of partial melting, Journal of Structural Geology 24 (6-7) (2002) 1045 - 1053, micro structural Processes: A Special Issue in Honor of the Career Contributions of R.H. Vernon. doi:http://dx.doi.org/10.1016/S0191-8141(01)00090-6.

[43] Y. Deubelbeiss, B. Kaus, J. Connolly, Direct numerical simulation of two-phase flow: Effective rheology and flow patterns of particle suspensions, Earth and Planetary Science Letters 290 (1-2) (2010) 1 - 12. doi:http://dx.doi.org/10.1016/j.epsl.2009.11.041.

[44] K. Thøgersen, M. Dabrowski, A. Malthe-Sørenssen, Transient cluster formation in sheared non-brownian suspensions, Phys. Rev. E 93 (2016) 022611. doi:10.1103/PhysRevE.93.022611.

[45] R. Mosé, P. Siegel, P. Ackerer, G. Chavent, Application of the mixed hybrid finite element approximation in a groundwater flow model: Luxury or necessity?, Water Resources Research 30 (11) (1994) 3001-3012. doi:10.1029/94WR01786.

[46] C. T. Miller, C. N. Dawson, M. W. Farthing, T. Y. Hou, J. Huang, C. E. Kees, C. Kelley, H. P. Langtangen, Numerical simulation of water resources problems: Models, methods, and trends, Advances in Water Resources 51 (2013) 405 - 437, 35th Year Anniversary Issue. doi:http://dx.doi.org/10.1016/j.advwatres.2012.05.008.

[47] R. Walsh, C. McDermott, O. Kolditz, Numerical modeling of stress-permeability coupling in rough fractures, Hydrogeology Journal 16 (4) (2008) 613. doi:10.1007/s10040-007-0254-1.

[48] K. Su, J. Latham, D. Pavlidis, J. Xiang, F. Fang, P. Mostaghimi, J. R. Percival, C. C. Pain, M. D. Jackson, Multiphase flow simulation through porous media with explicitly resolved fractures, Geofluids 15 (4) (2015) 592-607. doi:10.1111/gfl.12129. 
[49] H. Hoteit, A. Firoozabadi, An efficient numerical model for incompressible two-phase flow in fractured media, Advances in Water Resources 31 (6) (2008) 891 - 905. doi:http://dx.doi.org/10.1016/j.advwatres.2008.02.004.

[50] A. Souche, M. Dabrowski, T. B. Andersen, Modeling thermal convection in supradetachment basins: example from western norway, Geofluids 14 (1) (2014) 58-74. doi: $10.1111 /$ gfl.12042.

[51] S. Geiger, T. Driesner, C. A. Heinrich, S. K. Matthäi, Multiphase thermohaline convection in the earth's crust: I. a new finite element - finite volume solution technique combined with a new equation of state for nacl-h2o, Transport in Porous Media 63 (3) (2006) 399-434. doi:10.1007/s11242-005-0108-z.

[52] J. Hasenclever, S. Theissen-Krah, L. H. Rupke, J. P. Morgan, K. Iyer, S. Petersen, C. W. Devey, Hybrid shallow on-axis and deep off-axis hydrothermal circulation at fast-spreading ridges, Nature 508 (7497) (2014) 508-512, letter.

[53] H. Diersch, O. Kolditz, Coupled groundwater flow and transport: 2. thermohaline and 3d convection systems, Advances in Water Resources 21 (5) (1998) 401 - 425. doi:http://dx.doi.org/10.1016/S0309-1708(97)00003-1.

[54] S. Geiger, S. Roberts, S. Matthäi, C. Zoppou, A. Burri, Combining finite element and finite volume methods for efficient multiphase flow simulations in highly heterogeneous and structurally complex geologic media, Geofluids 4 (4) (2004) 284-299.

[55] T. Hantschel, A. I. Kauerauf, B. Wygrala, Finite element analysis and ray tracing modeling of petroleum migration, Marine and Petroleum Geology 17 (7) (2000) 815 - 820. doi:http://dx.doi.org/10.1016/S0264-8172(99)00061-6.

[56] B. Jung, G. Garven, J. R. Boles, Effects of episodic fluid flow on hydrocarbon migration in the newport-inglewood fault zone, southern california, Geofluids 14 (2) (2014) 234-250. doi:10.1111/gfl.12070.

[57] L. Wang, M. B. Cardenas, D. T. Slottke, R. A. Ketcham, J. M. Sharp, Modification of the local cubic law of fracture flow for weak inertia, tortuosity, and roughness, Water Resources Research 51 (4) (2015) 2064-2080. doi:10.1002/2014WR015815.

[58] B. Chareyre, A. Cortis, E. Catalano, E. Barthélemy, Pore-scale modeling of viscous flow and induced forces in dense sphere packings, Transport in Porous Media 92 (2) (2012) 473-493. doi:10.1007/s11242-011-9915-6.

[59] Y. Zaretskiy, S. Geiger, K. Sorbie, M. Förster, Efficient flow and transport simulations in reconstructed 3d pore geometries, Advances in Water Resources 33 (12) (2010) 1508 1516. doi:http://dx.doi.org/10.1016/j.advwatres.2010.08.008.

[60] G. Linga, J. Mathiesen, F. Renard, Self-similar distributions of fluid velocity and stress heterogeneity in a dissolving porous limestone, Journal of Geophysical Research: Solid Earth (2017) n/a-n/a2016JB013536. doi:10.1002/2016JB013536.

[61] T. Arbogast, D. S. Brunson, A computational method for approximating a darcy-stokes system governing a vuggy porous medium, Computational Geosciences 11 (3) (2007) 207218. doi:10.1007/s10596-007-9043-0. 
[62] M. Krotkiewski, I. Ligaarden, K.-A. Lie, D. Schmid, On the importance of the stokesbrinkman equations for computing effective permeability in karst reservoirs, Communications in Computational Physics 10 (2011) 1315-1332.

[63] R. Holme, D. H. Rothman, Lattice-gas and lattice-boltzmann models of miscible fluids, Journal of Statistical Physics 68 (3) (1992) 409-429. doi:10.1007/BF01341756.

[64] E. G. Flekkøy, U. Oxaal, J. Feder, T. Jøssang, Hydrodynamic dispersion at stagnation points: Simulations and experiments, Physical Review E 52 (5) (1995) 4952-4962. doi:10.1103/PhysRevE.52.4952.

[65] D. S. Clague, B. D. Kandhai, R. Zhang, P. M. A. Sloot, Hydraulic permeability of (un)bounded fibrous media using the lattice Boltzmann method, Phys. Rev. E 61 (1) (2000) 616-625.

[66] R. Tsay, S. Weinbaum, Viscous flow in a channel with periodic cross-bridging fibres: exact solutions and Brinkman approximation, Journal of Fluid Mechanics 226 (1991) 125-148. doi:10.1017/S0022112091002318.

[67] J. Fernandez, P. Kurowski, L. Limat, P. Petitjeans, Wavelength selection of fingering instability inside Hele-Shaw cells, Physics of Fluids 13 (2001) 3120-3125. doi:10.1063/1.1410120.

[68] P. Grosfils, J. P. Boon, J. Chin, E. S. Boek, Structural and dynamical characterization of Hele-Shaw viscous fingering, Philosophical Transactions of the Royal Society of London A: Mathematical, Physical and Engineering Sciences 362 (1821) (2004) 1723-1734. doi:10.1098/rsta.2004.1398.

[69] M. Venturoli, E. S. Boek, Two-dimensional lattice-Boltzmann simulations of single phase flow in a pseudo two-dimensional micromodel, Physica A: Statistical Mechanics and its Applications 362 (1) (2006) 23-29. doi:10.1016/j.physa.2005.09.006.

[70] D. P. Hong, C. Byon, Analytic correlation for the capillary pressure of micro-square-pillar arrays, International Journal of Precision Engineering and Manufacturing 15 (12) (2014) $2677-2680$.

[71] P. Horgue, F. Augier, P. Duru, M. Prat, M. Quintard, Experimental and numerical study of two-phase flows in arrays of cylinders, Chemical Engineering Science 102 (2013) 335-345.

[72] M. Nagel, F. Gallaire, Boundary elements method for microfluidic two-phase flows in shallow channels, Computers \& Fluids 107 (2015) 272-284.

[73] A. Laleian, A. Valocchi, C. Werth, An Incompressible, Depth-Averaged Lattice Boltzmann Method for Liquid Flow in Microfluidic Devices with Variable Aperture, Computation 3 (4) (2015) 600-615. doi:10.3390/computation3040600.

[74] M. Dabrowski, M. Krotkiewski, D. Schmid, Milamin: Matlab-based finite element method solver for large problems, Geochemistry, Geophysics, Geosystems 9 (4).

[75] Ł. Łaniewski-Wołłk, J. Rokicki, Adjoint Lattice Boltzmann for topology optimization on multi-GPU architecture, Computers \& Mathematics with Applications 71 (3) (2016) 833848. doi:10.1016/j.camwa.2015.12.043.

[76] M. Dzikowski, Ł. Łaniewski-Wołłk, J. Rokicki, Single Component Multiphase Lattice Boltzmann Method for Taylor/Bretherton Bubble Train Flow Simulations, Communications in Computational Physics 19 (04) (2016) 1042-1066. doi:10.4208/cicp.220115.110915a. 
[77] J. Feder, Random sequential adsorption, Journal of Theoretical Biology 87 (2) (1980) 237254.

[78] P. Prestininzi, A. Montessori, M. La Rocca, S. Succi, Reassessing the single relaxation time Lattice Boltzmann method for the simulation of Darcy's flows, International Journal of Modern Physics C 27 (04) (2016) 1650037. doi:10.1142/S0129183116500376. URL http://www.worldscientific.com/doi/abs/10.1142/S0129183116500376

[79] J. R. Shewchuk, Triangle: Engineering a 2d quality mesh generator and Delaunay triangulator, in: M. C. Lin, D. Manocha (Eds.), Applied Computational Geometry Towards Geometric Engineering, no. 1148 in Lecture Notes in Computer Science, Springer Berlin Heidelberg, 1996, pp. 203-222, dOI: 10.1007/BFb0014497.

[80] H. Si, Tetgen, a delaunay-based quality tetrahedral mesh generator, ACM Transactions on Mathematical Software (TOMS) 41 (2) (2015) 11.

[81] M. Krotkiewski, M. Dabrowski, MUTILS - a set of efficient modeling tools for multi-core CPUs implemented in MEX, in: EGU General Assembly Conference Abstracts, Vol. 15 of EGU General Assembly Conference Abstracts, 2013, pp. EGU2013-7877.

[82] M. Dabrowski, M. Krotkiewski, D. W. Schmid, MILAMIN: MATLAB-based finite element method solver for large problems, Geochemistry, Geophysics, Geosystems 9 (4) (2008) Q04030. doi:10.1029/2007GC001719.

[83] P. L. Bhatnagar, E. P. Gross, M. Krook, A Model for Collision Processes in Gases. I. Small Amplitude Processes in Charged and Neutral One-Component Systems, Physical Review 94 (3) (1954) 511-525. doi:10.1103/PhysRev.94.511.

[84] Z. Guo, C. Shu, Lattice Boltzmann Method and Its Applications in Engineering, Vol. 3 of Advances in Computational Fluid Dynamics, WORLD SCIENTIFIC, 2013.

[85] D. D'Humières, I. Ginzburg, M. Krafczyk, P. Lallemand, L.-S. Luo, Multiple-relaxationtime lattice Boltzmann models in three dimensions, Philosophical Transactions of the Royal Society A: Mathematical, Physical and Engineering Sciences 360 (1792) (2002) 437-451. doi:10.1098/rsta.2001.0955.

[86] M. Bernaschi, S. Succi, H. Chen, Accelerated Lattice Boltzmann Schemes for SteadyState Flow Simulations, Journal of Scientific Computing 16 (2) (2001) 135-144. doi:10.1023/A:1012230722915.

[87] R. Verberg, A. J. C. Ladd, Simulation of low-Reynolds-number flow via a time-independent lattice-Boltzmann method, Physical Review E 60 (3) (1999) 3366-3373. doi:10.1103/PhysRevE.60.3366.

[88] M. Geier, M. Schönherr, A. Pasquali, M. Krafczyk, The cumulant lattice Boltzmann equation in three dimensions: Theory and validation, Computers \& Mathematics with Applications 70 (4) (2015) 507-547. doi:10.1016/j.camwa.2015.05.001.

[89] A. Kupershtokh, Incorporating a body force term into the lattice Boltzmann equation. (In Russian), Vestnik NGU Series: Math., Mech. and Informatics 4 (2) (2004) 75-96.

[90] A. Kupershtokh, D. Medvedev, D. Karpov, On equations of state in a lattice Boltzmann method, Computers \& Mathematics with Applications 58 (5) (2009) 965-974. doi:10.1016/j.camwa.2009.02.024. 
[91] G. Falcucci, S. Ubertini, S. Succi, Lattice Boltzmann simulations of phase-separating flows at large density ratios: the case of doubly-attractive pseudo-potentials, Soft Matter 6 (18) (2010) 4357. doi:10.1039/c002974b.

URL http://xlink.rsc.org/?DOI=c002974b

[92] G. Falcucci, S. Ubertini, D. Chiappini, S. Succi, Modern lattice Boltzmann methods for multiphase microflows, IMA Journal of Applied Mathematics 76 (5) (2011) 712-725. doi:10.1093/imamat/hxr014.

URL https://academic.oup.com/imamat/article-lookup/doi/10.1093/imamat/hxr014

[93] Q. Zou, X. He, On pressure and velocity boundary conditions for the lattice Boltzmann BGK model, Physics of Fluids 9 (6) (1997) 1591-1598. doi:doi:10.1063/1.869307.

[94] M. Krotkiewski, M. Dabrowski, MUTILS - a set of efficient modeling tools for multi-core CPUs implemented in MEX, in: EGU General Assembly Conference Abstracts, Vol. 15 of EGU General Assembly Conference Abstracts, 2013, pp. EGU2013-7877.

[95] H. Brinkman, A calculation of the viscous force exerted by a flowing fluid on a dense swarm of particles, Applied Scientific Research 1 (1) (1947) 27-34.

[96] H. C. Brinkman, A calculation of the viscous force exerted by a flowing fluid on a dense swarm of particles, Flow, Turbulence and Combustion 1 (1) (1949) 27. doi:10.1007/BF02120313.

[97] M. Krotkiewski, I. S. Ligaarden, K.-A. Lie, D. W. Schmid, On the importance of the stokes-brinkman equations for computing effective permeability in karst reservoirs, Communications in Computational Physics 10 (5) (2011) 1315-1332.

[98] N. Martys, D. P. Bentz, E. J. Garboczi, Computer simulation study of the effective viscosity in brinkman's equation, Physics of Fluids (1994-present) 6 (4) (1994) 1434-1439.

[99] I. Pop, P. Cheng, Flow past a circular cylinder embedded in a porous medium based on the brinkman model, International Journal of Engineering Science 30 (2) (1992) 257-262.

[100] B. W. Thompson, Secondary flow in a Hele-Shaw cell, Journal of Fluid Mechanics 31 (1968) 379-395. doi:10.1017/S0022112068000212.

[101] L. Spielman, S. L. Goren, Model for predicting pressure drop and filtration efficiency in fibrous media, Environmental Science \& Technology 2 (4) (1968) 279-287.

[102] A. Chernyakov, The viscous drag of a rough cylinder in a liquid flow in a porous medium, Colloid Journal 63 (4) (2001) 499-505.

[103] C. Wang, Darcy-brinkman flow with solid inclusions, Chemical Engineering Communications 197 (3) (2010) 261-274.

[104] J. S. Lee, Y. C. Fung, Stokes flow around a circular cylindrical post confined between two parallel plates, Journal of Fluid Mechanics 37 (4) (1969) 657-670. doi:10.1017/S0022112069000796. 